\title{
MERCADO DE PROFESORES EN EL SISTEMA ESCOLAR URBANO CHILENO
}

\section{Introducción}

Durante los últimos años, numerosas investigaciones han relevado la importancia de los docentes como un factor determinante en el desempeño académico y la formación de habilidades blandas en los estudiantes (Sanders y Rivers, 1996; Rockoff, 2004; Barber y Mourshed, 2007). A pesar de lo anterior, en el mundo existe escasa información relativa a las dinámicas de oferta y demanda de profesores (OCDE, 2005).

La demanda de docentes en cada sistema educativo depende principalmente de tres factores: la matrícula de alumnos, la razón de alumnos por profesor y las tasas de retiro y jubilación de los docentes. Cada uno de estos factores puede variar al interior de cada sistema educativo según el nivel de enseñanza analizado. La oferta de profesores, a su vez, depende de variados factores entre los que se encuentra el número de nuevos profesionales, la proporción de estos que ingresan al sistema a ejercer la profesión, el stock acumulado de profesores y las tasas de retiro de los docentes. Más indirectamente, aunque de forma relevante, se incluyen factores como los salarios, las condiciones laborales, las exigencias para el ejercicio de la profesión y la valoración social de la profesión (Cooper y Alvarado, 2006).

La investigación disponible distingue principalmente entre sistemas educativos que presentan escasez de docentes y aquellos que se caracterizan por una sobreoferta de profesores. Los primeros (como Australia, Bélgica, Estados Unidos, Reino Unido y Suecia) enfrentan desafíos asociados al reclutamiento de profesores en materias específicas (típicamente matemática, ciencias e idiomas) o a su falta para zonas geográficas alejadas de los centros urbanos (OCDE, 2005). Para lidiar con los problemas que implica la escasez de profesores, los administradores educacionales han utilizado principalmente tres estrategias: a) contratar profesores menos 
calificados; b) asignar profesores especializados en un área específica para suplir las necesidades en otra diferente que presenta escasez; y c) utilizar profesores sustitutos. A nivel de sistema, el problema se ha enfrentado aumentando los incentivos económicos para el ejercicio de la profesión y realizando campañas de valoración social de la profesión (Cooper et al., 2006; Vegas, 2007).

El grupo de países que presenta sobreoferta de profesores es menos numeroso y ha sido escasamente investigado. Corea del Sur, Grecia y Chile forman parte de este grupo. Los principales retos asociados a esta situación consisten en la consecuente desvalorización social de la profesión, dificultades para atraer a los alumnos más talentosos a la carrera de Pedagogía y eventuales bajas en la calidad de la formación ofrecida (OCDE, 2005).

La experiencia internacional muestra casos de países que han enfrentado esta situación estableciendo barreras para el ingreso a la profesión docente. Algunas de estas han consistido en la fijación de cuotas para estudiantes en las carreras de educación, la restricción en el número de entidades formadoras y el aumento en los requisitos de ingreso para el estudio de la carrera. Adicionalmente, se suma el uso de exámenes de habilitación profesional y los procesos de certificación continua (Ingersoll, 2007).

Debido a la evidencia que sustenta la importancia del factor docente en el aprendizaje de los estudiantes, las investigaciones relativas al mercado laboral de profesores no se han limitado solo a estimar la cantidad requerida en los sistemas educativos. De hecho, parte importante de la investigación ha centrado su atención en la disponibilidad de profesores de calidad, utilizando principalmente medidas de desempeño en el aula o de calidad de la formación inicial para abordar este concepto (Santiago, 2002; Hanushek, 2011).

En Chile el principal referente de estimación de oferta y demanda de profesores es un estudio desarrollado por Montoya y Blackburn para el periodo 2004-2015. Esta investigación, sin embargo, estima horas docentes requeridas y no aborda la cantidad de profesores demandada en el periodo analizado. 
El presente trabajo analiza el stock y demanda de los docentes de zonas urbanas con el objetivo de entregar elementos para una reflexión que sustente políticas públicas relacionadas con la disponibilidad de profesores. Para ello, este artículo se estructura en cinco secciones: en la primera se describe la metodología utilizada para realizar las estimaciones; en la segunda se detalla la situación actual de la demanda docente para el sector escolar urbano chileno y se entrega información acerca del actual stock de docentes disponible para satisfacer dicha demanda. En un tercer apartado se presentan proyecciones del stock de profesores para los próximos ocho años. La cuarta sección, a su vez, describe la relación entre la proyección de la demanda y el stock de profesores, detallando su evolución por zonas geográficas, niveles y subsectores. La quinta parte se enfoca en realizar estimaciones sobre la base de ciertas políticas públicas de reciente discusión en el país. Finalmente, se presentan las principales conclusiones y recomendaciones que se extraen del análisis antes mencionado.

\section{Metodología}

A continuación se presentan algunas consideraciones metodológicas utilizadas en las secciones III y IV correspondientes a la proyección de la demanda y stock de docentes, respectivamente.

En este artículo, la demanda docente corresponde al número de profesores de aula en establecimientos urbanos del sistema de educación formal, que son requeridos para cubrir las horas básicas del currículo nacional para cada nivel y subsector. Para obtener esta cifra se realizó una estimación de la evolución de la matrícula de estudiantes para los próximos años. Para ello se consideraron datos administrativos del Mineduc de 2011, diferenciados por nivel educativo (g), región $(\mathrm{r})$, dependencia (d) y año (t). Considerando esto, la matrícula se entiende como el resultado de cuatro factores: la tasa de natalidad (que determina el ingreso a primero básico) y las tasas de reprobación, 
retiro y deserción del sistema ${ }^{3}$. Junto con esta información, se toma como referencia el currículo actual (incorporando la modificación proyectada de este para 2017) y se proyecta la cantidad de cursos que existirán para cada nivel, dependencia, región y año (división entre matrícula y el número promedio de alumnos proyectado).

Del cálculo anterior se obtiene el número de horas pedagógicas sobre la base de la cual se estima la cantidad de docentes requeridos para cada nivel y subsector de enseñanza en los establecimientos educacionales en el periodo 2012-2020, según la evolución de la matrícula de estudiantes. La conversión de horas a docentes corresponde a la división del total de horas requeridas para cada nivel/especialidad por el promedio de horas que trabaja actualmente un profesor de aula de un establecimiento urbano para cada una de dichas especialidades. Para esto último se utilizó el promedio de contratación cronológica a la semana por especialidad, designando el $75 \%$ a clases en aula y el resto a planificación ${ }^{4}$.

Con la finalidad de determinar cuál será el stock de profesores que existirá anualmente en cada nivel-subsector para responder al requerimiento de docentes (demanda), se utilizó como base las cifras de 2011. Así, las proyecciones incluyeron la tasa de retiro de docentes en ejercicio (3,011\%), que está compuesta por la tasa de retiro de docentes y la tasa de jubilación ${ }^{5}$. Dicha tasa se calculó en función de los datos disponibles en la Encuesta Longitudinal Docente, entre 2005 y 2009. De esta forma, el dato abarcaba un periodo de cinco años. Para registrar las variaciones anuales se optó por transformar la tasa tetraanual simple a una tasa interanual compuesta:

3 De esta manera, la tasa de matrícula para el primer año puede describirse como: Matrícula

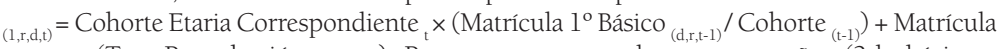

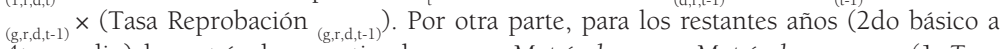
4to medio) la matrícula se entiende como: Matrícula ${ }_{(g, r, d, t)}=$ Matrícula $_{(g-1, r, d, t,-1)} \times(1-$ Tasa

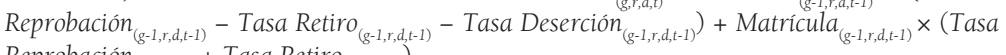

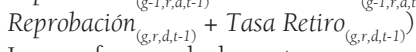

4 Los profesores declaran tener un contrato de 33 horas (Encuesta Longitudinal Docente 2009). Adicionalmente, el Ministerio de Educación señala que la relación entre horas de aula y horas de planificación es 3/4.

5 Este dato fue construido sobre la base de la cifra que entrega la Encuesta Longitudinal Docente 2009 


\section{TASA TETRA-ANUAL $=(\sqrt[4]{1+12,6 \%})-1$}

Este cálculo ajusta anualmente la cifra de profesores en ejercicio, pero sin afectar al número de docentes recién titulados que se incorporan al sistema cada año.

Para modelar la evolución de titulados en carreras de educación, se utilizó una función logarítmica que toma como referencia los registros de los titulados entre 2007 y 2011 por especialidad y que proyecta su crecimiento hasta 2020. Para incorporar la cifra de nuevos profesores que ingresan anualmente a ejercer a los establecimientos escolares se utilizó una tasa de participación basada en los datos de "Mi Futuro", en el estudio de Montoya (2010). Esta tasa se mantiene constante durante el periodo considerado para la proyección y es diferenciada por especialidad y nivel de enseñanza. Esta cifra corresponde a la proporción de nuevos docentes que ingresan a ejercer en el sistema anualmente. Sumada a la cifra de profesores en el sistema (afectada por la tasa de retiro) para cada año proyectado se obtiene el stock de docentes.

De la comparación entre el stock de profesores para cada año y el número de docentes requeridos en el mismo periodo se desprende el déficit o superávit para cada nivel-subsector.

De manera adicional a los análisis de demanda y stock ya mencionados se presentan estimaciones que consideran modificaciones en la política educativa, las que, en caso de ser implementadas, podrían afectar los niveles de selectividad de la carrera de pedagogía y generar restricciones para el ejercicio de la profesión. Las consideraciones metodológicas utilizadas para llevar a cabo el análisis de cada uno de estos escenarios se explican en mayor detalle en las secciones respectivas.

6 "Mi Futuro" es un portal del Ministerio de Educación de Chile que entrega información sobre educación superior a estudiantes de enseñanza media. En particular entrega información sobre la duración y aranceles de carreras universitarias y técnicas, además de informar acerca de empleabilidad e ingresos promedio percibido por titulados de distintas disciplinas. 


\section{Proyecciones de la demanda de profesores}

El siguiente apartado muestra los datos de la proyección del comportamiento de la demanda de profesores del sistema educativo chileno. Tal como se cita en estudios anteriores (Montoya y Blackburn, 2005) se genera una serie de supuestos que posibilita estimar el comportamiento presente y futuro del stock y demanda de profesores, permitiendo evaluar la capacidad del mercado de hacer confluir ambos elementos.

\subsection{Proyección de la matrícula escolar}

$\mathrm{Al}$ analizar los posibles cambios en la composición de la matrícula según nivel de enseñanza, se observa una disminución en educación básica y en el ciclo de enseñanza media para la modalidad científicohumanista. La reducción de la primera se explicaría, en parte importante, por la caída en la tasa de natalidad esperada y por la mantención de las tasas de deserción y repitencia de alumnos. La única modalidad de enseñanza en la que aumentaría la matrícula sería en la educación técnico profesional, con un incremento de poco más de $13 \%$.

Tabla 1: Proyección matrícula por nivel de enseñanza (miles de estudiantes)

\begin{tabular}{|l|c|c|c|c|c|c|c|c|c|c|}
\hline $\begin{array}{l}\text { Nivel de } \\
\text { enseñanza }\end{array}$ & 2012 & 2013 & 2014 & 2015 & 2016 & 2017 & 2018 & 2019 & 2020 & $\begin{array}{c}\text { Var 2012- } \\
2020\end{array}$ \\
\hline Ed. básica & 1.757 & 1.744 & 1.738 & 1.732 & 1.730 & 1.732 & 1.742 & 1.756 & 1.765 & $0,46 \%$ \\
\hline Ed. media HC & 741 & 728 & 709 & 692 & 678 & 661 & 642 & 622 & 610 & $-17,69 \%$ \\
\hline Ed. media TP & 189 & 199 & 202 & 205 & 208 & 212 & 214 & 215 & 214 & $13,02 \%$ \\
\hline TOTAL & 2.688 & 2.671 & 2.649 & 2.629 & 2.616 & 2.605 & 2.598 & 2.592 & 2.590 & $-3,66 \%$ \\
\hline
\end{tabular}

Fuente: Elaboración propia a partir de datos Mineduc.

Ahora bien, al proyectar la evolución de la matrícula según la dependencia de los establecimientos, se manifiesta la misma tendencia que otros estudios han mostrado: un aumento sostenido y progresivo del sector particular subvencionado, en desmedro del sector municipal (Elacqua, 2012). Según el modelo de estimación utilizado, se observaría una caída de 3,66\% en el total de matriculados entre 2012 y el 2020, reflejando la tasa de natalidad esperada para los próximos años. 
162 MERCADO DE PROFESORES EN EL SISTEMA ESCOLAR URBANO CHILENO M. Sánchez, G. Gutiérrez, H. Hochschild, M. Medeiros, M. Ortiz, M. Sepúlveda

Tabla 2: Proyección de matrícula según dependencia (miles de estudiantes)

\begin{tabular}{|l|c|c|c|c|c|c|c|c|c|c|}
\hline $\begin{array}{l}\text { Dependencia } \\
\text { administrativa }\end{array}$ & 2012 & 2013 & 2014 & 2015 & 2016 & 2017 & 2018 & 2019 & 2020 & $\begin{array}{c}\text { Var 2012- } \\
2020\end{array}$ \\
\hline Municipal & 1.061 & 1.045 & 1.026 & 1.008 & 991 & 973 & 960 & 950 & 944 & $-11,07 \%$ \\
\hline $\begin{array}{l}\text { Particular } \\
\text { subvencionada }\end{array}$ & 1.415 & 1.419 & 1.417 & 1.415 & 1.420 & 1.426 & 1.431 & 1.435 & 1.438 & $1,58 \%$ \\
\hline Particular pagada & 211 & 208 & 206 & 206 & 206 & 206 & 207 & 207 & 208 & $-1,53 \%$ \\
\hline TOTAL & 2.688 & 2.671 & 2.649 & 2.629 & 2.616 & 2.605 & 2.598 & 2.592 & 2.590 & $-3,66 \%$ \\
\hline
\end{tabular}

Fuente: Elaboración propia a partir de datos Mineduc.

Junto con esto, parece relevante observar la distribución de matrícula según las zonas geográficas, ya que tanto la demanda como el stock de docentes tiene una movilidad que está limitada por la extensión geográfica del país. Para ello se han definido tres macrozonas geográficas: a) zona norte, que incorpora las regiones de Arica y Parinacota, Tarapacá, Antofagasta, Atacama y Coquimbo; b) zona centro, que incluye las regiones de Valparaíso, Metropolitana, Libertador Bernardo O'Higgins, Maule y Biobío; y c) zona sur, que incluye las regiones de La Araucanía, Los Ríos, Los Lagos, Aysén, Magallanes y la Antártica Chilena. Es posible observar que la zona sur presentaría la mayor disminución de la matrícula escolar en el periodo estimado, registrando $17 \%$ menos de alumnos desde el 2012 al 2020. La zona centro es la más importante en términos de matrícula, captando más del $70 \%$ del total y manteniendo su magnitud con los años, registrándose una pequeña variación negativa, pero no significativa. Por último, la zona norte con casi $15 \%$ de la matrícula del país, muestra una pequeña variación positiva con los años.

Tabla 3: Proyección de matrícula escolar por macrozona geográfica (miles de estudiantes)

\begin{tabular}{|l|c|c|c|c|c|c|c|c|c|c|}
\hline Macrozona & 2012 & 2013 & 2014 & 2015 & 2016 & 2017 & 2018 & 2019 & 2020 & Var 2012-2020 \\
\hline Norte & 360 & 363 & 363 & 363 & 363 & 363 & 363 & 364 & 365 & $1,26 \%$ \\
\hline Centro & 1.977 & 1.968 & 1.956 & 1.946 & 1.941 & 1.938 & 1.936 & 1.935 & 1.936 & $-2,11 \%$ \\
\hline Sur & 350 & 341 & 331 & 320 & 312 & 305 & 299 & 294 & 289 & $-17,51 \%$ \\
\hline TOTAL & 2.688 & 2.671 & 2.649 & 2.629 & 2.616 & 2.605 & 2.598 & 2.592 & 2.590 & $-3,66 \%$ \\
\hline
\end{tabular}

Fuente: Elaboración propia a partir de datos Mineduc. 


\subsection{Proyección cantidad de cursos}

En general, la proyección muestra que se produciría una disminución de la cantidad de cursos en todos los niveles de enseñanza, estimándose una reducción total aproximada de 3.000 cursos entre 2012 y 2020, lo que equivale a una baja del 3,9\% durante el periodo analizado. La cantidad de cursos refleja la disminución de la matrícula, afectando principalmente a aquellos científico-humanista en favor del aumento de cursos de carácter técnico profesional. El nivel básico mantendría casi constante el número de cursos. En concordancia con las tendencias proyectadas para la matrícula escolar según dependencia, se observa una marcada disminución de la cantidad de cursos del sector municipal y un crecimiento para el sector particular subvencionado.

Por último, al clasificar la matrícula por macrozona se observaría cómo la notable reducción de esta en la zona sur tiene directa relación con la disminución de cursos en la misma área.

\subsection{Horas docentes requeridas}

El Ministerio de Educación ha proyectado cambios en el currículo de los diferentes niveles de enseñanza, los que conllevan modificaciones en la cantidad de horas lectivas de ciertas asignaturas. El cambio proyectado en los ciclos de enseñanza (contenido en la Ley General de Educación) ${ }^{7}$ implicará que tanto el nivel básico como el medio constarán de seis años. Este cambio se concretará en 2017, año en el que disminuirán los requerimientos del sistema de docentes de enseñanza básica, pues $7^{\circ}$ y $8^{\circ}$ básico pasarán a formar parte del ciclo de enseñanza media. Junto con esto, aumentarían los requerimientos de la mayoría de las especialidades de enseñanza media.

En términos generales se observa una disminución en la proyección de la cantidad de horas docentes requeridas a medida que avanza el tiempo.

7 Más información en http://www.leychile.cl/Navegar?idNorma=1006043. 


\subsection{Docentes requeridos}

El siguiente gráfico muestra una proyección de la demanda de profesores para el área urbana del sistema entre 2012 y $2020^{8}$. Considerando los supuestos mencionados en la metodología y manteniendo las condiciones actuales en nuestro sistema, se observaría una disminución creciente en la cantidad de docentes requeridos hasta 2020.

Figura 1: Proyección de demanda de profesores 2011-2020

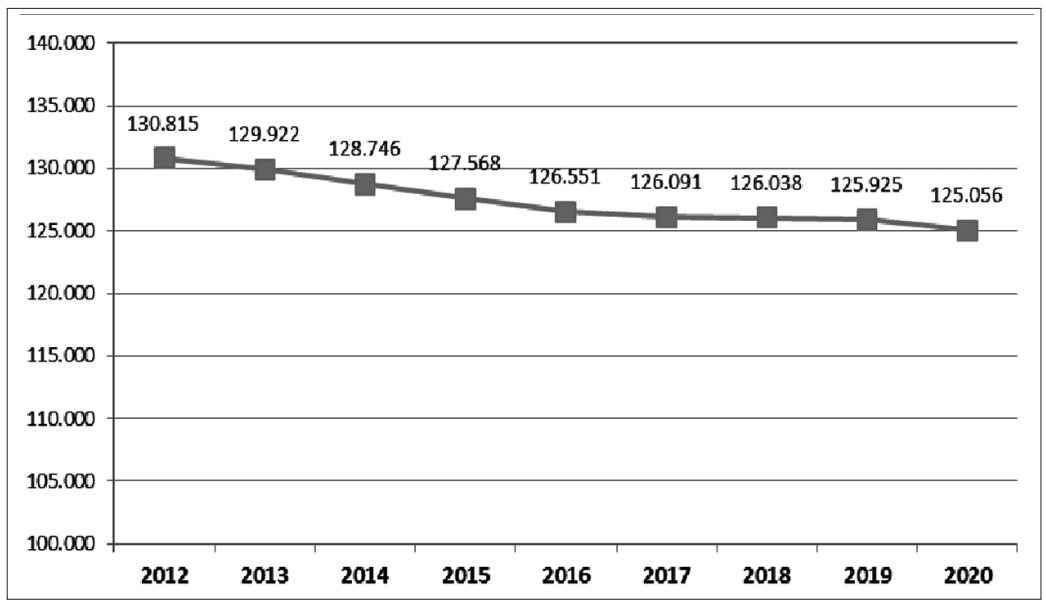

Fuente: Elaboración propia a partir de datos de la Unidad de Estudios Mineduc 2012.

Si se analiza esta proyección según la dependencia de los establecimientos, se observará una disminución de la demanda docente en el sector municipal. Los profesores requeridos en establecimientos privados aumentarían levemente, pero este aumento no es equivalente a la disminución del sector municipal. De esta manera, es posible inferir que la disminución de la demanda proyectada se originaría por la reducción en la cantidad de docentes que se requerirían en establecimientos municipales, sector que ha presentado una tendencia a la disminución, tanto de establecimientos como de matrículas asociadas.

8 Los totales son mayores a los presentados por especialidad, pues incorporan aquellas que no son analizadas de manera independiente como educación tecnológica. 
Figura 2: Proyección demanda docente según dependencia 2011-2020

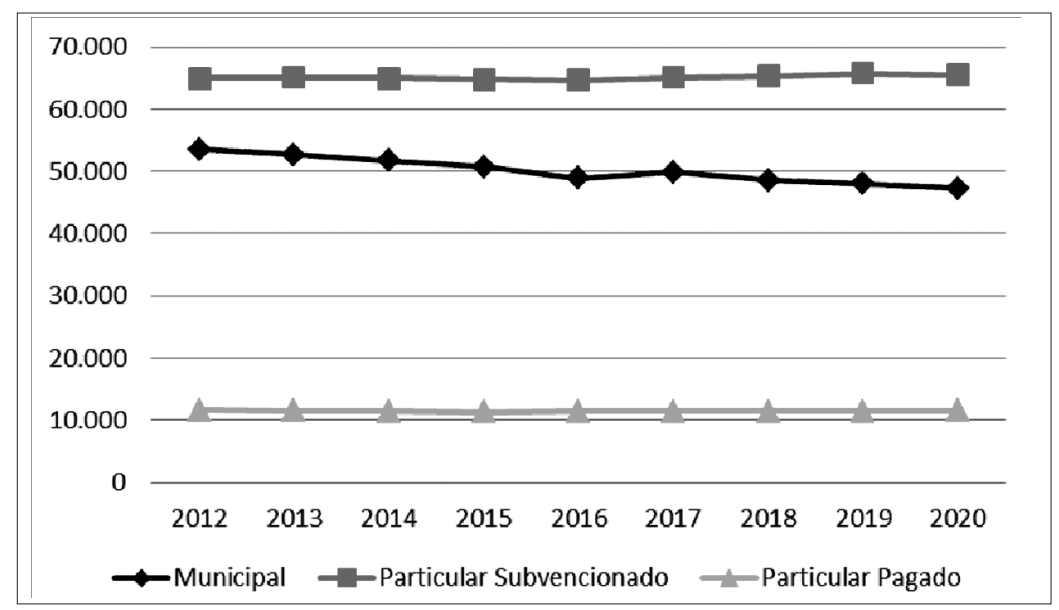

Fuente: Elaboración propia a partir de datos Unidad de Estudios Mineduc 2012.

A su vez se realizó una estimación del número de docentes requeridos en el país según el nivel/especialidad en el que realizan clases. Para esta labor se estableció un nuevo supuesto de estimación. Según la base de Idoneidad Docente de $2011^{9}$ se calculó el promedio de horas cronológicas de contratación de los profesores de aula urbanos, diferenciados por especialidad y se dividió el número de horas requeridas por especialidad por el promedio de horas de cada una de estas. Con lo anterior se hizo posible corregir, en parte, la sobreestimación que pudiese afectar a las especialidades de enseñanza media para las que los docentes suelen ser contratados por menos horas, o la subestimación en el caso de los profesores de enseñanza básica que usualmente son contratados por jornada completa.

A modo de síntesis, se puede señalar que según las estimaciones, en los próximos años el requerimiento de profesores sería menor que el actual en la mayoría de los niveles, especialidades, dependencias y zonas, dando cuenta de un proceso de contracción de la demanda.

9 Base de datos generada por el Mineduc, que presenta información de todos los docentes que se encuentran trabajando en establecimientos educacionales del país, asociada al establecimiento en el que realizan la mayor cantidad de horas. 
166 MERCADO DE PROFESORES EN EL SISTEMA ESCOLAR URBANO CHILENO M. Sánchez, G. Gutiérrez, H. Hochschild, M. Medeiros, M. Ortiz, M. Sepúlveda

Tabla 4: Estimación del número de docentes requeridos por especialidad en el sistema educacional

\begin{tabular}{|c|c|c|c|c|c|c|c|c|c|}
\hline Demanda por tipo de profesor & 2012 & 2013 & 2014 & 2015 & 2016 & 2017 & 2018 & 2019 & 2020 \\
\hline General básica & 47.183 & 47.108 & 47.341 & 47.685 & 47.863 & 43.308 & 43.447 & 43.518 & 43.566 \\
\hline General media & 11.935 & 11.718 & 11.371 & 11.036 & 10.556 & 16.054 & 15.658 & 15.434 & 15.409 \\
\hline Matemática - básica & 5.614 & 5.501 & 5.392 & 5.252 & 5.192 & 2.619 & 2.662 & 2.677 & 2.683 \\
\hline Lenguaje y Comunicación - básica & 5.614 & 5.501 & 5.392 & 5.252 & 5.192 & 2.619 & 2.662 & 2.677 & 2.683 \\
\hline Matemáticas - media & 4.437 & 4.404 & 4.327 & 4.257 & 4.184 & 6.452 & 6.400 & 6.427 & 6.318 \\
\hline Lenguaje y Comunicación - media & 4.437 & 4.404 & 4.327 & 4.257 & 4.184 & 6.453 & 6.400 & 6.427 & 6.319 \\
\hline $\begin{array}{l}\text { Estudio y Comprensión de la } \\
\text { Naturaleza }\end{array}$ & 4.492 & 4.400 & 4.313 & 4.201 & 4.154 & 2.095 & 2.129 & 2.142 & 2.146 \\
\hline $\begin{array}{l}\text { Estudio y Comprensión de la } \\
\text { Sociedad }\end{array}$ & 4.492 & 4.400 & 4.313 & 4.201 & 4.154 & 2.095 & 2.129 & 2.142 & 2.146 \\
\hline Biología & 1.768 & 1.735 & 1.688 & 1.643 & 1.579 & 2.494 & 2.438 & 2.421 & 2.409 \\
\hline Física & 1.767 & 1.735 & 1.687 & 1.643 & 1.579 & 2.494 & 2.437 & 2.421 & 2.409 \\
\hline Química & 1.767 & 1.735 & 1.687 & 1.643 & 1.579 & 2.493 & 2.437 & 2.420 & 2.408 \\
\hline Historia & 4.444 & 4.428 & 4.347 & 4.270 & 4.191 & 6.003 & 5.969 & 5.945 & 5.848 \\
\hline Idioma & 6.476 & 6.388 & 6.266 & 6.129 & 6.012 & 6.271 & 6.230 & 6.238 & 6.168 \\
\hline Arte & 5.709 & 5.600 & 5.481 & 5.339 & 5.224 & 4.065 & 4.035 & 4.027 & 4.019 \\
\hline Religión & 6.240 & 6.171 & 6.107 & 6.044 & 5.973 & 5.860 & 5.823 & 5.815 & 5.807 \\
\hline Filosofía & 995 & 979 & 932 & 882 & 807 & 815 & 771 & 679 & 714 \\
\hline Educación Física & 7.284 & 7.221 & 7.171 & 7.128 & 7.066 & 6.955 & 6.920 & 6.913 & 6.906 \\
\hline Educación Técnico Profesional & 5.946 & 6.272 & 6.362 & 6.440 & 6.754 & 6.643 & 7.158 & 7.218 & 6.740 \\
\hline TOTAL & 130.600 & 129.699 & 128.503 & 127.302 & 126.242 & 125.787 & 125.704 & 125.540 & 124.698 \\
\hline
\end{tabular}

Fuente: Elaboración propia a partir de datos Mineduc.

\section{Proyección del stock de profesores}

En la presente sección se proyecta el stock de profesores para el sistema escolar chileno en los próximos ocho años.

\subsection{Proyección de titulados en carreras de educación}

En caso de mantenerse las condiciones actuales en el sistema de Formación Inicial Docente, en todas las carreras se proyectaría un aumento significativo en la cantidad de titulados para los próximos años. Se estima que en 2020 se incrementaría la cantidad general de titulados en $16 \%$ respecto del año 2010. El menor aumento porcentual de titulados para 2020 respecto del 2012 lo tendrían las carreras de Filosofía y Educación Básica con -4\% y 7\%, respectivamente. Por otro lado, Física, Química e Inglés serían las carreras con un mayor aumento porcentual de titulados superando el 30\% entre 2012 y 2020. 


\subsection{Docentes disponibles}

La mantención de las condiciones ya mencionadas provocaría un aumento significativo en la cantidad de docentes disponibles para todos los años, hasta 2020.

Figura 3: Proyección de stock de profesores 2012-2020

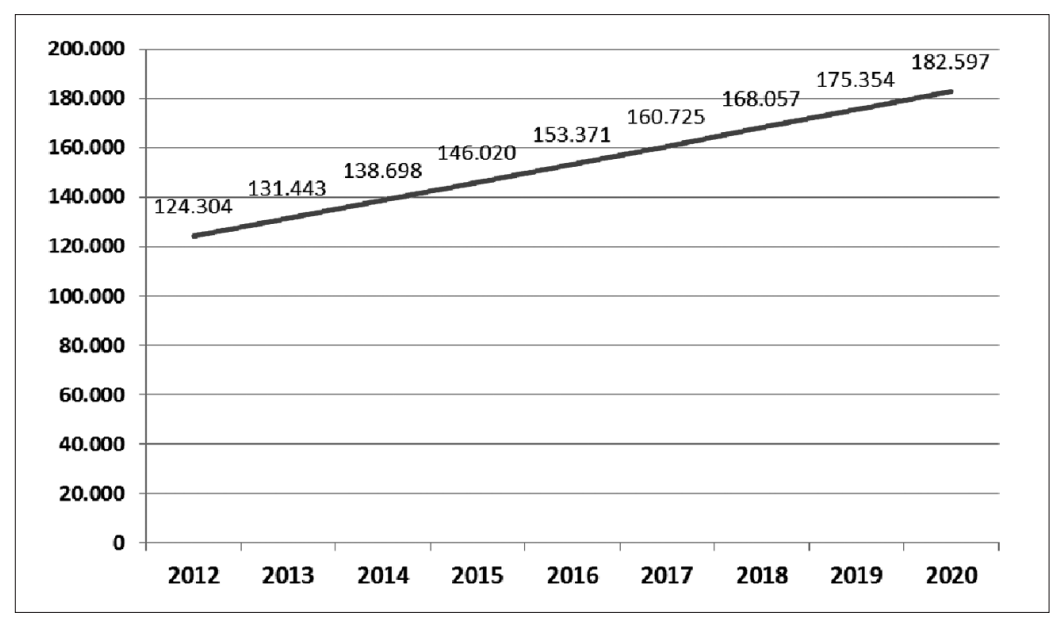

Fuente: Elaboración propia en base a datos Mineduc 2012.

Si dicha proyección es segmentada por especialidad, es posible observar que se presentaría un marcado aumento en la cantidad de docentes de Educación Básica e incrementos de distintas magnitudes en las diferentes especialidades.

\section{Relación entre el stock y la demanda de profesores en Chile}

Con el fin de analizar el comportamiento del mercado docente se estiman proyecciones del escenario actual, en las cuales se detalla el posible comportamiento futuro del stock y de la demanda docente si se mantuvieran vigentes las condiciones de nuestro sistema educacional. 
Para comparar ambas tendencias se consideró la demanda docente para Educación General Básica ${ }^{10}$ y la de la mayoría de las especialidades de enseñanza media.

Al analizar el mercado docente a nivel nacional es posible observar que el stock general de docentes iría en alza durante el periodo estimado, mientras que los requerimientos del mercado tenderían a la baja. De este modo, la brecha entre ambos grupos aumentaría a medida que avanza dicho periodo.

Figura 4: Proyección del mercado docente 2012-2020

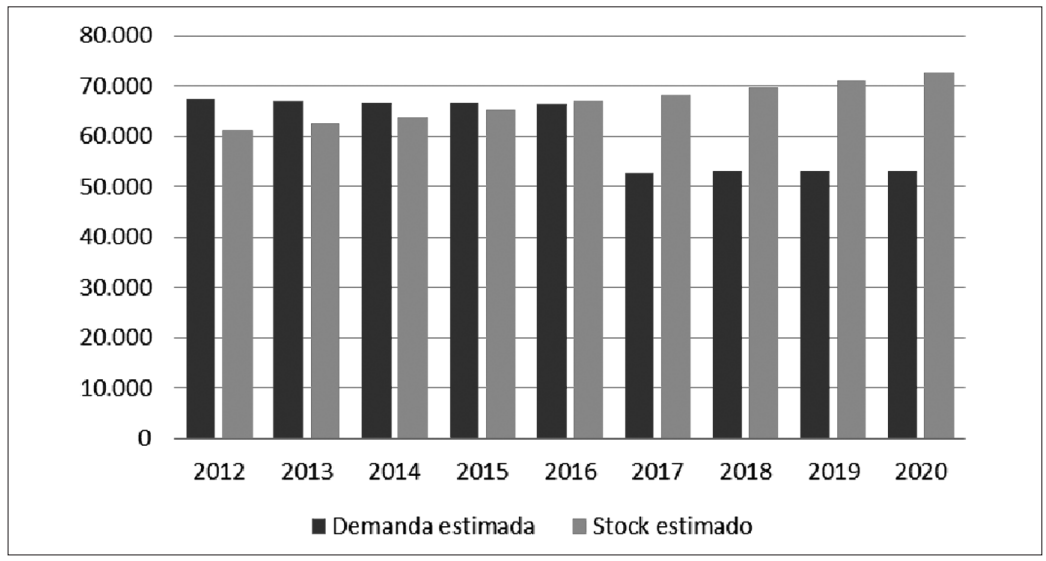

Fuente: Elaboración propia a partir de datos Unidad de Estudios Mineduc, SIES e Idoneidad Docente.

\subsection{Según especialidad}

Al analizar el mercado docente según nivel/especialidad para 2012, se observa un superávit de docentes para todas las especialidades, con excepción de Educación General Básica y Física. En el caso de esta última asignatura se requeriría un aumento del nivel de titulados con la finalidad de responder al 2,3\% de demanda insatisfecha de dicha especialidad. En Educación General Básica, en tanto, existiría 6\% de demanda insatisfecha. Del resto de las especialidades destacan, por el

10 Este grupo considera a los docentes requeridos para educación general básica, matemática de primero a octavo básico, lenguaje de primero a octavo básico, estudio y comprensión de la sociedad y de la naturaleza de primero a octavo básico, educación tecnológica de primero a octavo básico y educación artística de primero a cuarto básico. 
elevado exceso de oferta que presentan, Biología, Filosofía y Lenguaje, con $122 \%, 80 \%$ y $76 \%$, respectivamente.

Tabla 5: Brechas entre demanda y stock de profesores por especialidad 2012

\begin{tabular}{|l|c|r|r|c|}
\hline Nivel/Especialidad & Stock 2012 & Demanda 2012 & Stock-Demanda & $\begin{array}{c}\text { Diferencia porcentual sobre } \\
\text { la demanda }\end{array}$ \\
\hline Ed. Básica & 63.118 & 67.395 & -4.276 & $-6,35 \%$ \\
\hline Lenguaje & 7.823 & 4.437 & 3.386 & $76,33 \%$ \\
\hline Matemática & 6.886 & 4.437 & 2.449 & $55,19 \%$ \\
\hline Física & 1.727 & 1.767 & -41 & $-2,31 \%$ \\
\hline Química & 2.446 & 1.767 & 678 & $38,39 \%$ \\
\hline Biología & 3.922 & 1.768 & 2.154 & $121,86 \%$ \\
\hline Filosofía & 1.792 & 995 & 797 & $80,07 \%$ \\
\hline Historia & 7.362 & 4.444 & 2.919 & $65,68 \%$ \\
\hline Ed. Artística & 5.804 & 5.709 & 94 & $1,65 \%$ \\
\hline Inglés & 8.622 & 6.476 & 2.146 & $33,13 \%$ \\
\hline Ed. Física & 9.495 & 7.284 & 2.211 & $30,35 \%$ \\
\hline
\end{tabular}

Fuente: Elaboración propia a partir de datos Unidad de Estudios Mineduc, SIES e Idoneidad Docente.

Al proyectar el comportamiento del mercado docente hasta 2020, sería posible observar que las especialidades de física y educación básica lograrían suplir su brecha de demanda en 2013. Para el 2016 se observaría un superávit para todas las especialidades. Con el cambio curricular de 2017, física volvería a presentar una escasez de oferta docente, tendencia que se mantendría hasta 2020. Con dicha modificación curricular, algunas especialidades de educación media (lenguaje, matemática, ciencias e historia) presentarían reducciones de las brechas de stock, mientras que lo contrario ocurriría con educación básica.

Sin embargo, aun con los nuevos requisitos curriculares, se observa para 2020 un superávit, mayor al 55\% de los requerimientos del sistema, para todas las especialidades, exceptuando física y química. En particular al 2020 las especialidades de inglés y educación física presentarían 200\% más de la demanda necesaria en el sistema escolar ${ }^{11}$.

11 El caso de educación artística merece un análisis individual, pues incorpora las especialidades de artes plásticas y música. Lo anterior hace que no puedan extraerse conclusiones muy certeras de este grupo específico, pues no es posible observar el comportamiento de ambas especialidades independientemente. Por otro lado las horas de música deberían ser cubiertas por docentes titulados en Educación Musical y las de Artes Plásticas por docentes titulados en Artes Visuales, sin poder intercambiar la función de ambos, lo que hace que estas se comporten como dos especialidades independientes. 
170 MERCADO DE PROFESORES EN EL SISTEMA ESCOLAR URBANO CHILENO -

M. Sánchez, G. Gutiérrez, H. Hochschild, M. Medeiros, M. Ortiz, M. Sepúlveda

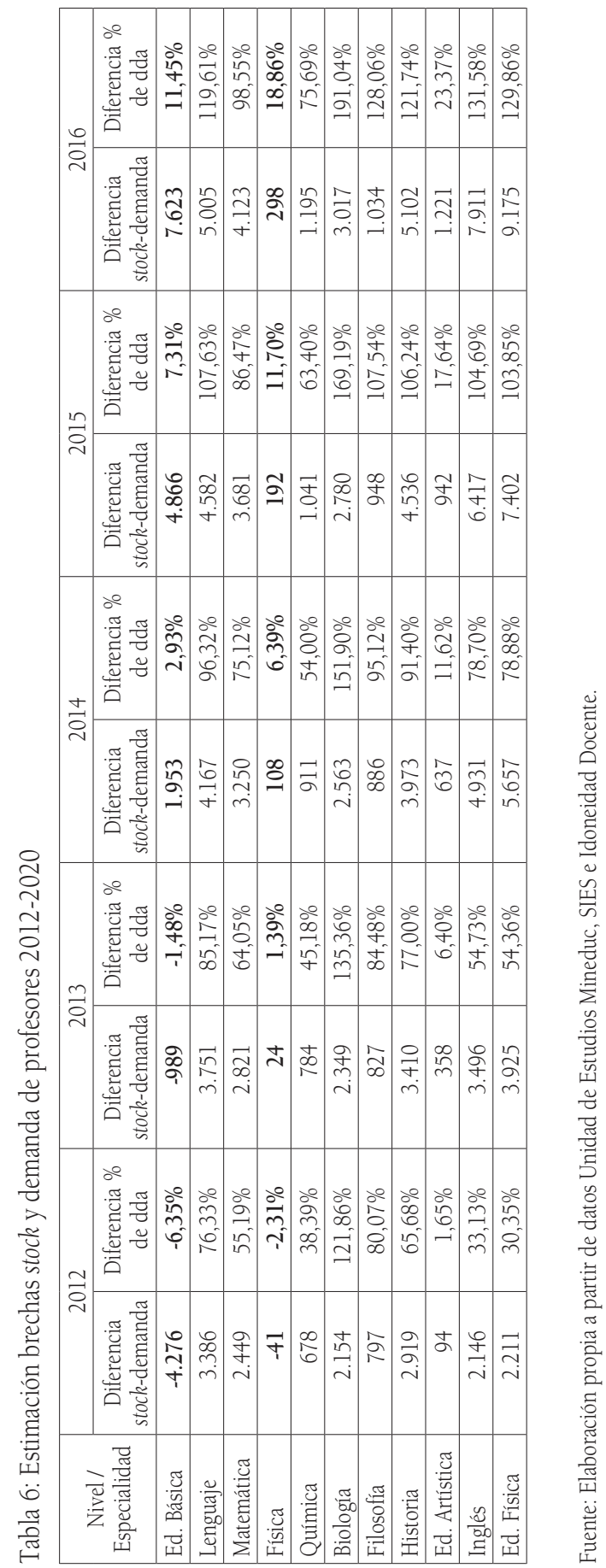




\subsection{Según especialidad y macrozona geográfica}

El comportamiento del stock y la demanda docente difiere significativamente en las distintas macrozonas geográficas. Al proyectar el comportamiento según ubicación geográfica, se observa que la zona norte presentaría superávit de docentes en todas los niveles/especialidades, con excepción de física (que presentaría un déficit en todos los años estimados), educación básica (que presentaría déficit hasta 2017) y filosofía (que presentaría déficit en los primeros años estimados). La zona centro mostraría tendencias similares, con diferencias en física, que no presentaría déficit hasta la implementación de cambio curricular en 2017. La zona sur por su parte, no mostraría déficit de docentes de educación básica, pero presentaría un déficit de docentes de física de mayor importancia relativa en comparación con otras especialidades de la zona.

Junto con lo anterior, merece especial atención el notable superávit de docentes de inglés y educación física en las tres zonas geográficas, que iría en constante aumento durante el periodo proyectado. 
172 MERCADO DE PROFESORES EN EL SISTEMA ESCOLAR URBANO CHILENO -

M. Sánchez, G. Gutiérrez, H. Hochschild, M. Medeiros, M. Ortiz, M. Sepúlveda

Tabla 7: Estimación brechas entre demanda y stock de profesores por especialidad y macrozona, zona norte 2012-2020

\begin{tabular}{|l|r|r|r|r|r|r|r|r|c|}
\hline Especialidad & \multicolumn{1}{c|}{2012} & \multicolumn{1}{c}{2013} & \multicolumn{1}{c}{2014} & \multicolumn{1}{|c|}{2015} & \multicolumn{1}{c}{2016} & \multicolumn{1}{c}{2017} & 2018 & 2019 & 2020 \\
\hline Ed. Básica & $-8,21 \%$ & $-6,61 \%$ & $-5,76 \%$ & $-4,87 \%$ & $-4,36 \%$ & $21,37 \%$ & $20,73 \%$ & $20,98 \%$ & $21,63 \%$ \\
\hline Lenguaje & $78,03 \%$ & $83,13 \%$ & $91,77 \%$ & $100,34 \%$ & $110,37 \%$ & $42,07 \%$ & $47,70 \%$ & $51,91 \%$ & $54,86 \%$ \\
\hline Matemática & $62,97 \%$ & $66,09 \%$ & $72,31 \%$ & $78,36 \%$ & $85,59 \%$ & $24,24 \%$ & $28,07 \%$ & $30,63 \%$ & $32,11 \%$ \\
\hline Física & $-24,05 \%$ & $-23,76 \%$ & $-21,64 \%$ & $-19,29 \%$ & $-15,48 \%$ & $-46,64 \%$ & $-44,78 \%$ & $-44,11 \%$ & $-43,63 \%$ \\
\hline Química & $41,50 \%$ & $49,52 \%$ & $61,46 \%$ & $74,32 \%$ & $90,85 \%$ & $25,66 \%$ & $35,28 \%$ & $42,07 \%$ & $48,37 \%$ \\
\hline Biología & $147,54 \%$ & $158,20 \%$ & $174,62 \%$ & $191,68 \%$ & $213,91 \%$ & $103,11 \%$ & $114,89 \%$ & $121,82 \%$ & $127,75 \%$ \\
\hline Filosofía & $-7,44 \%$ & $-3,05 \%$ & $6,73 \%$ & $18,67 \%$ & $38,36 \%$ & $41,46 \%$ & $54,24 \%$ & $82,59 \%$ & $78,74 \%$ \\
\hline Historia & $60,35 \%$ & $67,52 \%$ & $78,66 \%$ & $90,01 \%$ & $103,17 \%$ & $49,46 \%$ & $56,47 \%$ & $64,40 \%$ & $67,94 \%$ \\
\hline Ed. Artística & $1,37 \%$ & $4,91 \%$ & $9,28 \%$ & $14,64 \%$ & $20,75 \%$ & $58,80 \%$ & $62,50 \%$ & $65,83 \%$ & $69,72 \%$ \\
\hline Inglés & $63,61 \%$ & $87,69 \%$ & $114,36 \%$ & $142,66 \%$ & $173,06 \%$ & $184,70 \%$ & $208,82 \%$ & $231,52 \%$ & $251,93 \%$ \\
\hline Ed. Física & $21,10 \%$ & $40,22 \%$ & $59,86 \%$ & $80,10 \%$ & $100,85 \%$ & $124,63 \%$ & $145,87 \%$ & $166,23 \%$ & $186,71 \%$ \\
\hline
\end{tabular}

Fuente: Elaboración propia a partir de datos Unidad de Estudios Mineduc, SIES e Idoneidad Docente.

Tabla 8: Estimación brechas entre demanda y stock de profesores por especialidad y macrozona, zona centro 2012-2020

\begin{tabular}{|l|r|r|r|r|r|r|c|c|c|}
\hline Especialidad & \multicolumn{1}{|c|}{2012} & \multicolumn{1}{|c|}{2013} & 2014 & \multicolumn{1}{|c|}{2015} & 2016 & 2017 & 2018 & 2019 & 2020 \\
\hline Ed. Básica & $-8,42 \%$ & $-4,40 \%$ & $-0,40 \%$ & $3,24 \%$ & $6,47 \%$ & $27,88 \%$ & $29,80 \%$ & $31,79 \%$ & $33,66 \%$ \\
\hline Lenguaje & $74,30 \%$ & $45,47 \%$ & $48,50 \%$ & $51,24 \%$ & $53,90 \%$ & $31,35 \%$ & $34,29 \%$ & $36,09 \%$ & $39,53 \%$ \\
\hline Matemática & $52,25 \%$ & $37,67 \%$ & $41,22 \%$ & $44,41 \%$ & $47,52 \%$ & $21,92 \%$ & $25,34 \%$ & $27,45 \%$ & $31,41 \%$ \\
\hline Física & $5,09 \%$ & $8,89 \%$ & $13,32 \%$ & $17,51 \%$ & $22,63 \%$ & $-18,89 \%$ & $-13,63 \%$ & $-10,34 \%$ & $-7,22 \%$ \\
\hline Química & $42,90 \%$ & $33,42 \%$ & $37,08 \%$ & $40,52 \%$ & $44,59 \%$ & $15,42 \%$ & $19,69 \%$ & $22,51 \%$ & $25,15 \%$ \\
\hline Biología & $111,84 \%$ & $55,54 \%$ & $58,32 \%$ & $60,86 \%$ & $63,72 \%$ & $44,84 \%$ & $47,78 \%$ & $49,72 \%$ & $51,51 \%$ \\
\hline Filosofía & $108,29 \%$ & $53,61 \%$ & $56,13 \%$ & $58,56 \%$ & $62,04 \%$ & $61,70 \%$ & $63,83 \%$ & $68,31 \%$ & $66,87 \%$ \\
\hline Historia & $67,90 \%$ & $44,58 \%$ & $48,79 \%$ & $52,42 \%$ & $55,71 \%$ & $39,44 \%$ & $42,57 \%$ & $45,14 \%$ & $48,63 \%$ \\
\hline Ed. Artística & $5,21 \%$ & $9,83 \%$ & $14,60 \%$ & $19,41 \%$ & $23,21 \%$ & $41,68 \%$ & $43,64 \%$ & $45,18 \%$ & $46,65 \%$ \\
\hline Inglés & $30,30 \%$ & $34,20 \%$ & $43,14 \%$ & $50,42 \%$ & $56,13 \%$ & $58,38 \%$ & $62,10 \%$ & $64,92 \%$ & $67,90 \%$ \\
\hline Ed. Física & $32,85 \%$ & $36,56 \%$ & $45,27 \%$ & $51,94 \%$ & $57,39 \%$ & $62,02 \%$ & $65,49 \%$ & $68,26 \%$ & $70,61 \%$ \\
\hline
\end{tabular}

Fuente: Elaboración propia a partir de datos Unidad de Estudios Mineduc, SIES e Idoneidad Docente. 
Tabla 9: Estimación brechas entre demanda y stock de profesores por especialidad y macrozona, zona sur 2012-2020

\begin{tabular}{|l|c|r|r|r|r|r|r|r|r|}
\hline Especialidad & 2012 & 2013 & 2014 & 2015 & 2016 & 2017 & 2018 & 2019 & 2020 \\
\hline Ed. Básica & $5,99 \%$ & $15,63 \%$ & $23,29 \%$ & $29,98 \%$ & $35,54 \%$ & $53,98 \%$ & $56,54 \%$ & $58,71 \%$ & $60,65 \%$ \\
\hline Lenguaje & $45,82 \%$ & $48,88 \%$ & $52,63 \%$ & $56,07 \%$ & $58,75 \%$ & $39,45 \%$ & $42,71 \%$ & $45,61 \%$ & $48,78 \%$ \\
\hline Matemática & $38,48 \%$ & $44,35 \%$ & $50,59 \%$ & $56,07 \%$ & $60,40 \%$ & $44,13 \%$ & $49,10 \%$ & $53,41 \%$ & $57,63 \%$ \\
\hline Física & $-25,24 \%$ & $-22,03 \%$ & $-16,22 \%$ & $-10,32 \%$ & $-4,19 \%$ & $-61,51 \%$ & $-52,77 \%$ & $-45,68 \%$ & $-39,69 \%$ \\
\hline Química & $11,78 \%$ & $13,99 \%$ & $18,01 \%$ & $22,09 \%$ & $26,32 \%$ & $-14,39 \%$ & $-8,38 \%$ & $-3,52 \%$ & $0,54 \%$ \\
\hline Biología & $59,88 \%$ & $62,59 \%$ & $65,82 \%$ & $68,79 \%$ & $71,57 \%$ & $57,39 \%$ & $60,95 \%$ & $63,83 \%$ & $66,24 \%$ \\
\hline Filosofía & $10,56 \%$ & $6,84 \%$ & $9,04 \%$ & $13,10 \%$ & $19,31 \%$ & $18,78 \%$ & $22,48 \%$ & $29,58 \%$ & $23,39 \%$ \\
\hline Historia & $37,42 \%$ & $40,85 \%$ & $45,75 \%$ & $50,50 \%$ & $54,35 \%$ & $39,21 \%$ & $43,11 \%$ & $47,10 \%$ & $50,57 \%$ \\
\hline Ed. Artística & $-19,65 \%$ & $-18,61 \%$ & $-17,55 \%$ & $-15,84 \%$ & $-12,84 \%$ & $13,96 \%$ & $15,54 \%$ & $16,37 \%$ & $16,87 \%$ \\
\hline Inglés & $16,69 \%$ & $27,77 \%$ & $37,45 \%$ & $45,65 \%$ & $52,51 \%$ & $55,67 \%$ & $60,41 \%$ & $64,33 \%$ & $67,80 \%$ \\
\hline Ed. Física & $20,59 \%$ & $33,54 \%$ & $43,35 \%$ & $51,04 \%$ & $57,00 \%$ & $62,08 \%$ & $65,84 \%$ & $68,85 \%$ & $71,34 \%$ \\
\hline
\end{tabular}

Fuente: Elaboración propia a partir de datos Unidad de Estudios MINEDUC, SIES e Idoneidad Docente.

\section{Políticas que podrían modificar la estimación}

En la sección anterior se presentó un panorama general acerca del mercado docente en nuestro país. En el siguiente apartado se simulan algunos escenarios en los cuales la implementación de políticas públicas podrían modificar las estimaciones antes presentadas.

\subsection{Implementación de criterios de selectividad en el ingreso a carreras de educación}

La implementación de eventuales restricciones en el ingreso a las carreras de educación ha sido un tópico largamente discutido en círculos académicos y legislativos. A causa de la magnitud del número de egresados de las carreras de educación, la implementación de medidas de este tipo podría implicar modificaciones importantes para el stock de profesores en los próximos años.

Para realizar esta estimación se consideró conjuntamente las siguientes tres condiciones ${ }^{12}$ :

12 Los criterios mencionados fueron tomados del Proyecto de Ley que establece condiciones para la formación inicial docente y el ejercicio profesional (Boletín 8189-04), por ser la más reciente propuesta en la materia al momento de realizar esta investigación. 
a. Puntaje PSU promedio (lenguaje-matemáticas) mayor o igual a 550 puntos.

b. Pertenecer al 15\% superior del ranking de su establecimiento escolar.

c. Puntaje PSU promedio superior a 500 puntos promedio y pertenecer al 30\% superior del ranking de su establecimiento.

Se considerará un periodo de estimación del 2012 al 2025 con el fin de evaluar las posibles consecuencias de la implementación de criterios de selectividad a partir de 2014. Para ello se reproducen las estimaciones de demanda docente hasta 2025 y se asume la misma tasa de titulación del escenario actual hasta 2017, es decir, se mantienen las brechas estimadas anteriormente hasta 2018. Las restricciones en el ingreso de estudiantes tendrían efecto a partir de 2019, cuando la cantidad de titulados caería.

Por otra parte, solo se considera la matrícula en carreras de educación que cumplen con los filtros de selección y se realiza una proyección logarítmica de dicha matrícula. Junto con esto se consideran las tasas de retención de educación superior para carreras universitarias de cohorte 2010 y se utiliza el promedio de las mismas al quinto año para los dos niveles de interés: 69\% de retención promedio al quinto año de educación básica y $61 \%$ promedio en educación media. Se asumen las mismas tasas para institutos profesionales (Consejo Nacional de Educación). El cálculo anterior permite determinar la proyección de titulados para el periodo 2018 - 2024, los cuales se incorporan en el mercado el año posterior a su titulación.

Al desagregar los escenarios según subsectores de enseñanza, se observaría una tendencia al alza en el stock docente de educación básica alcanzando su máximo en 2018, para luego comenzar a disminuir levemente hasta el final del periodo. En cuanto al equilibrio de mercado se observaría una demanda insatisfecha, situación que permanecería hasta 2013, cuando se produciría un giro en la brecha de demanda que perduraría hasta el final de la estimación (2025). Al comparar los posibles resultados con la estimación base (escenario actual), se observaría una disminución del superávit acumulado, alcanzando de todas formas alrededor de 10.000 docentes en 2025. 
Figura 5: Comparación brechas stock y demanda educación básica, escenario base y con nuevos requisitos de selectividad

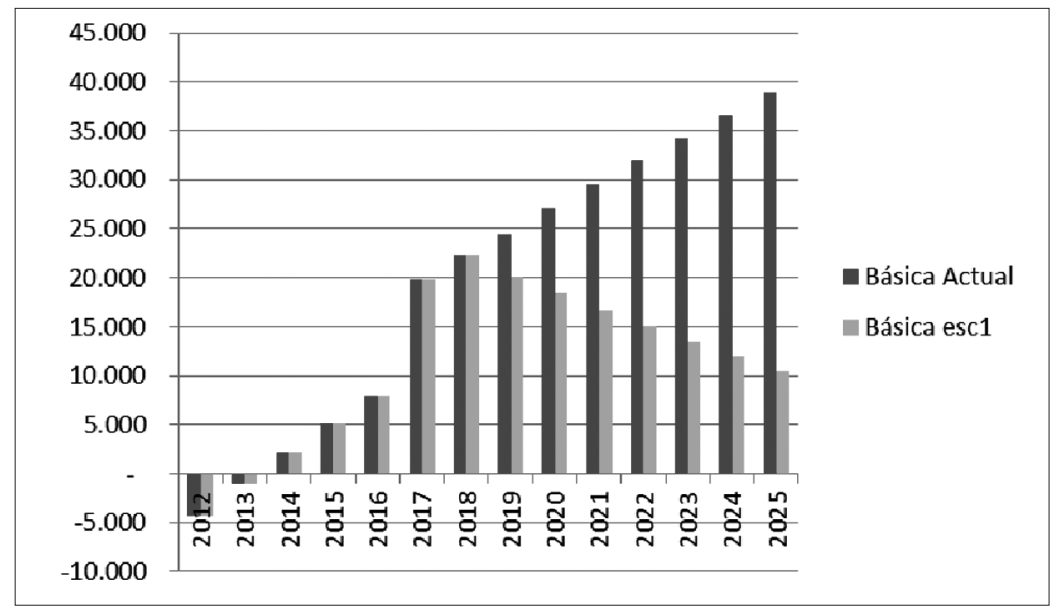

Fuente: Elaboración propia a partir de estimación Mineduc, SIES e Idoneidad Docente.

Para educación media se observaría un alza en la demanda estimada, aumentando su requerimiento tras la implementación del cambio curricular. Por el lado de la oferta, se observaría una tendencia creciente del stock docente que se estabilizaría a partir de 2018 en aproximadamente 85.000 docentes, lo anterior disminuiría la brecha de superávit docente en el agregado de las especialidades de educación media a partir de 2019.

Comparando las brechas existentes entre el escenario actual estimado y este nuevo escenario, se observaría un superávit agregado que se mantiene relativamente constante a partir de 2019 y hasta el final de la estimación. 
176 MERCADO DE PROFESORES EN EL SISTEMA ESCOLAR URBANO CHILENO M. Sánchez, G. Gutiérrez, H. Hochschild, M. Medeiros, M. Ortiz, M. Sepúlveda

Figura 6: Comparación brechas stock y demanda educación media, escenario base y con nuevos requisitos de selectividad

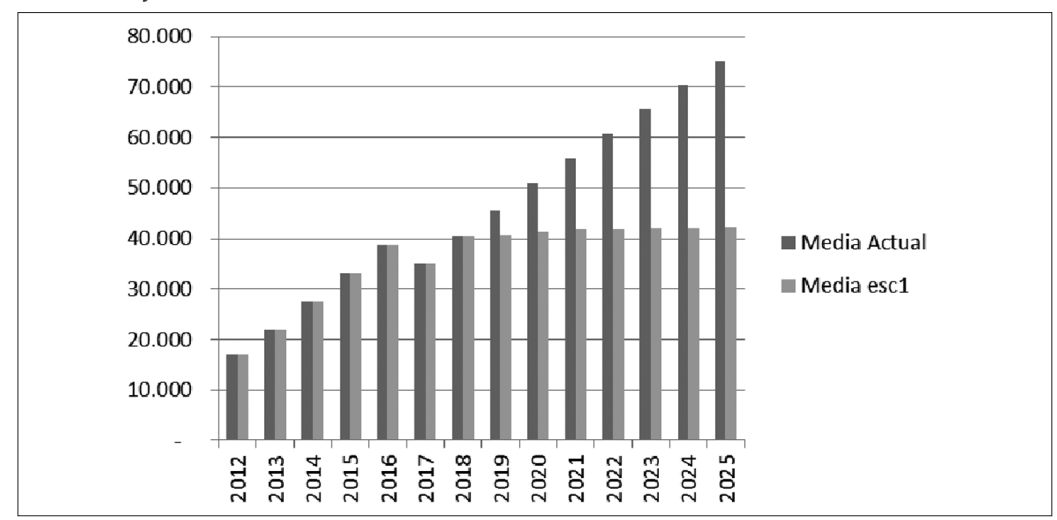

Fuente: Elaboración propia a partir de estimación Mineduc, SIES e Idoneidad Docente.

Al desagregar las estimaciones por macrozonas y comparar las posibles consecuencias de esta política por agregados regionales, se observaría que la zona norte presenta un déficit de docentes de educación básica que se mantiene hasta 2017. Luego, a partir de 2018 y bajo el supuesto de las restricciones estimadas en el escenario 1, comenzaría a disminuir el superávit de docentes llegando a ser negativo el 2015, y por ende representando un nuevo déficit.

Figura 7: Comparación brechas stock y demanda educación básica por macrozona, zona norte

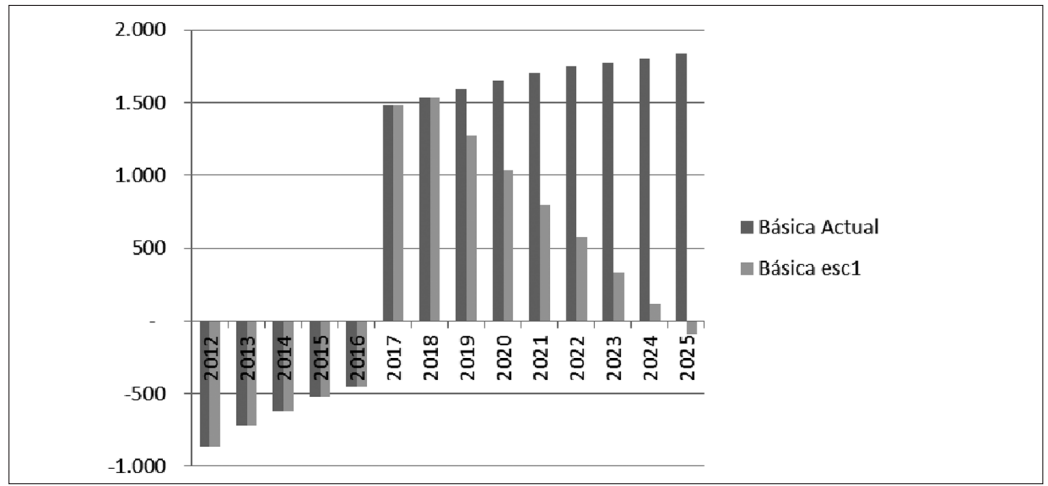

Fuente: Elaboración propia a partir de estimación Mineduc, SIES e Idoneidad Docente

Para el nivel de enseñanza media se observaría una disminución de la brecha de superávit a partir del año 2018, llegando a 4.000 para el año 2025. 
Figura 8: Comparación brechas stock y demanda educación media por macrozona, zona norte

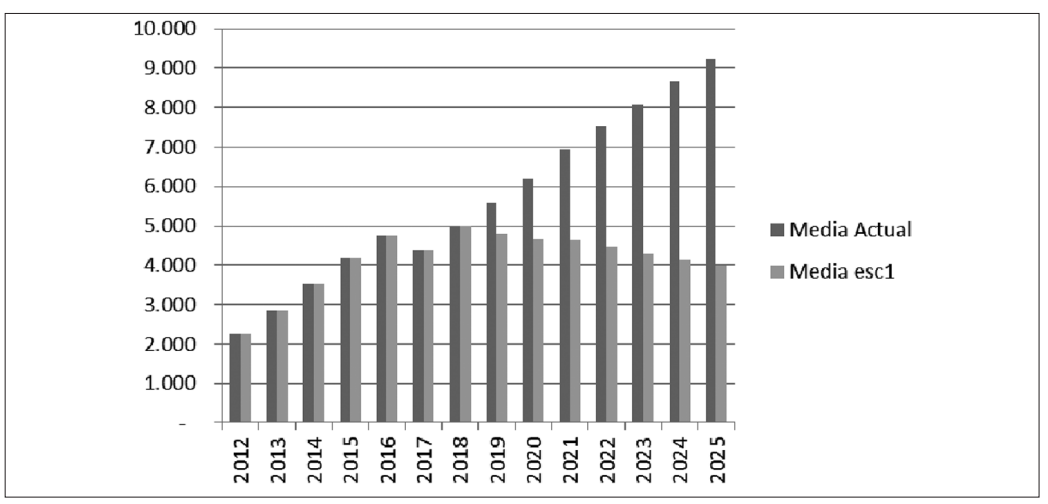

Fuente: Elaboración propia a partir de estimación Mineduc, SIES e Idoneidad Docente.

Lo anterior dista de la magnitud de superávit que se observaría a nivel nacional, siendo muchísimo menor, lo que podría dar cuenta de la tendencia de ingreso de estudiantes de bajo rendimiento a las carreras de pedagogías en la zona norte del país, y advierte la necesidad de considerar políticas de atracción específicas para esta zona, en caso de implementarse estas restricciones.

La macrozona centro presentaría una demanda insatisfecha de docentes de básica hasta el 2014. Luego, a partir del 2018 se observa que, de acuerdo con los nuevos criterios de selectividad, disminuiría el superávit de docentes en relación con el escenario actual, llegando alrededor de 10.000 para el año 2025.

Figura 9: Comparación brechas stock y demanda educación básica por macrozona, zona centro

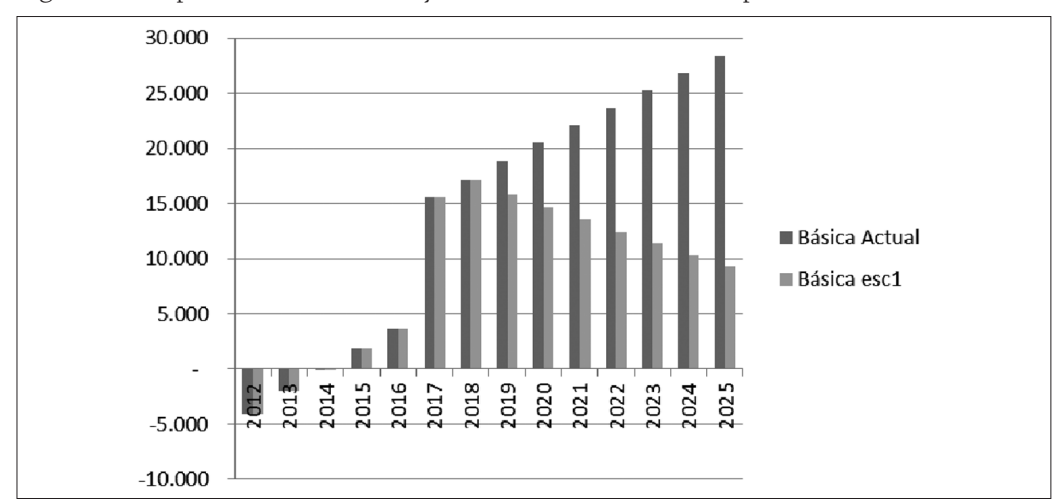

Fuente: Elaboración propia a partir de estimación Mineduc, SIES e Idoneidad Docente 
En cuanto a los docentes de media, el superávit estimado para el escenario actual se mantendría en ascenso, alcanzando una cifra superávit de aproximadamente 30.000 docentes en 2018. Posteriormente se reduciría la brecha bajo el escenario de simulación, pero se mantendría un superávit hasta el término del periodo.

Figura 10: Comparación brechas stock y demanda educación media por macrozona, zona centro

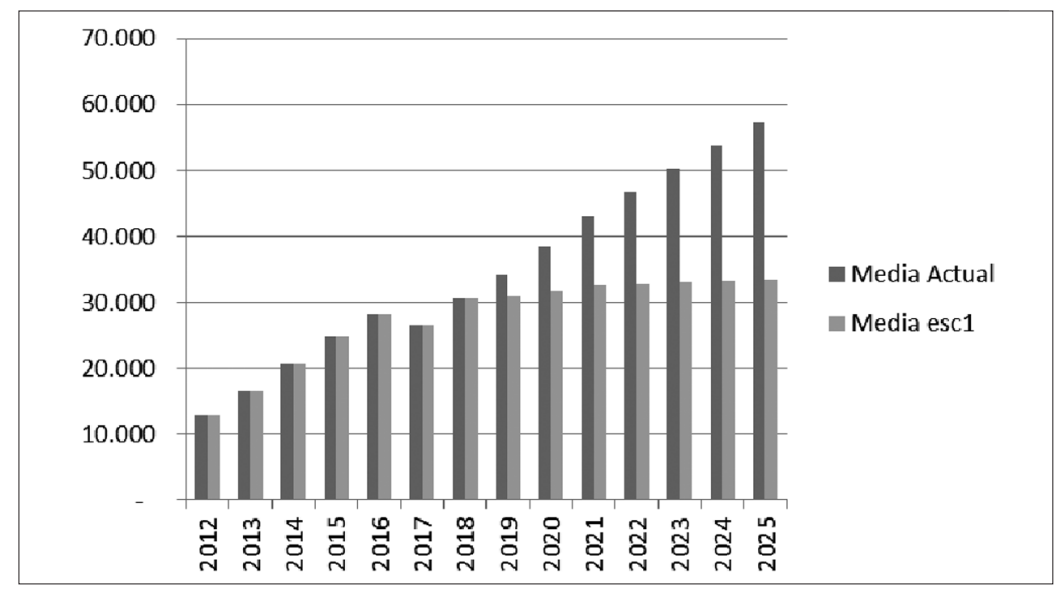

Fuente: Elaboración propia a partir de estimación Mineduc, SIES e Idoneidad Docente.

La macrozona sur, caracterizada por la presencia de un importante superávit de docentes de educación básica, el que se mantendría en constante ascenso bajo el escenario actual. Sin embargo, la simulación de las nuevas condiciones de entrada a la carrera afectaría esta situación, disminuyendo dicho superávit a partir del 2018, pero mostrando una leve tendencia al alza hasta el 2025 de aproximadamente 6.000 docentes. 
Figura 11: Comparación brechas stock y demanda educación básica por macrozona, zona sur

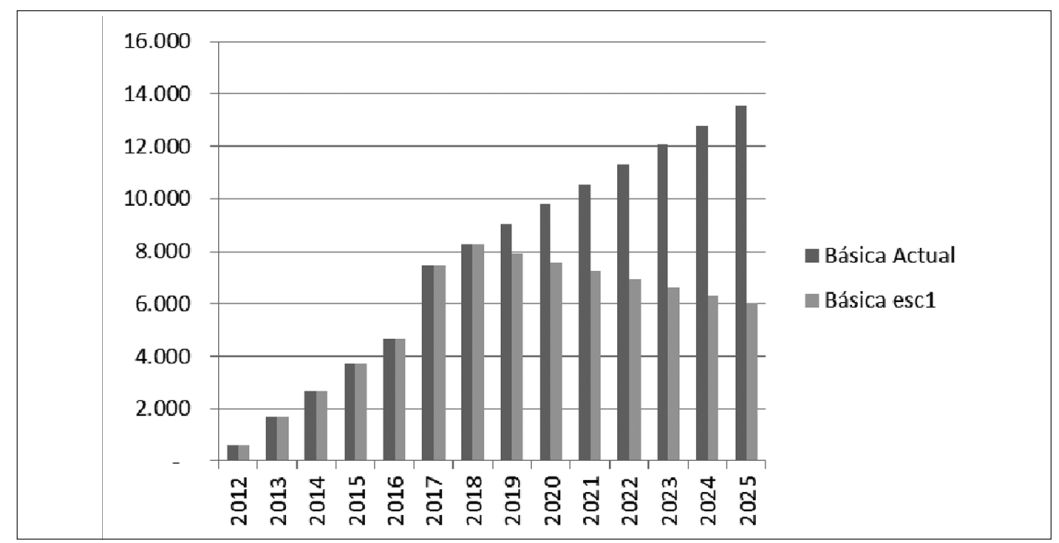

Fuente: Elaboración propia a partir de estimación Mineduc, SIES e Idoneidad Docente

Para los docentes de educación media se observaría una reducción en la brecha de superávit docente entre el 2016 y el 2017 , pero luego a partir del 2018 el superávit demostraría una tendencia al aumento bajo ambas estimaciones, aunque este sería menor bajo los nuevos criterios de ingreso a la carrera de Pedagogía.

Figura 12: Comparación brechas stock y demanda educación media por macrozona, zona sur

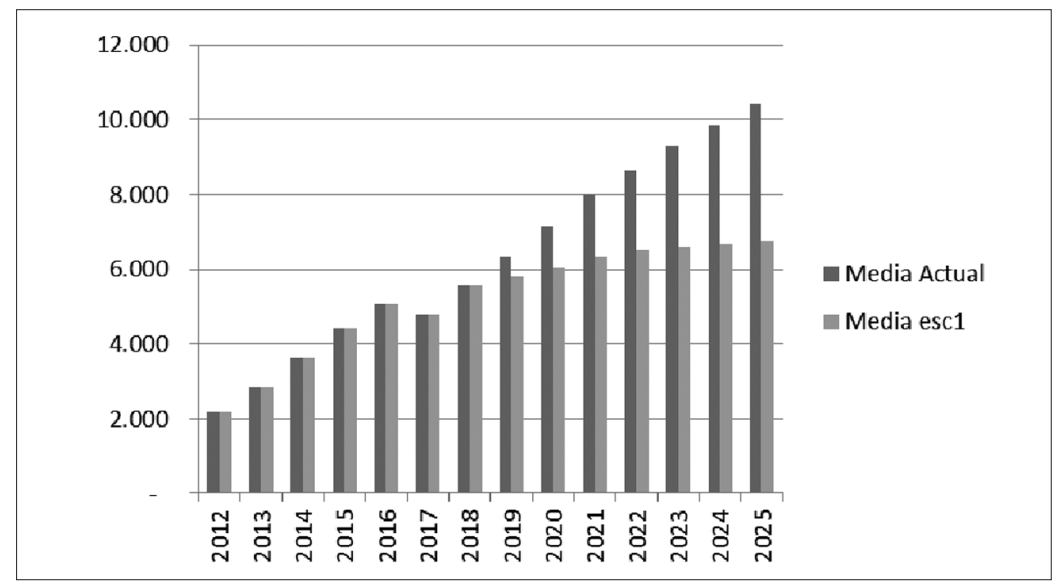

Fuente: Elaboración propia a partir de estimación Mineduc, SIES e Idoneidad Docente. 


\subsection{Selectividad al ingreso y aumento de horas no lectivas}

Las políticas públicas relativas a la profesión docente no solo consideran la atracción de talentos y la necesidad de aumentar la selectividad de ingreso a las carreras de educación, sino que también la importancia de mejorar las condiciones de trabajo. Como parte ello se discute la modificación de la proporción de horas no lectivas en la contratación docente. De acuerdo con lo anterior, se evalúan las implicancias sobre el mercado docente de la implementación conjunta de dichas políticas.

La estimación asume que el aumento de horas no lectivas entraría en vigencia a partir de 2014, incrementando el porcentaje de horas no lectivas en cinco puntos porcentuales, lo que modifica el supuesto respecto de las horas de contratación y aumenta el requerimiento docente a partir de dicho año. En cuanto a la selectividad de ingreso se mantienen los supuestos utilizados en el escenario anterior.

El mercado general de educación básica presentaría una brecha insatisfecha de docentes hasta 2014 si se consideran las nuevas restricciones de entrada. Bajo este mismo escenario, en 2015, la demanda y oferta se situarían en niveles muy similares, sin embargo, entre el 2016 y el 2018 la brecha iría en aumento para comenzar a descender hacia 2019 y continuar el descenso hasta 2025, año en el que el superávit sería levemente superior a 5.000 docentes. 
Figura 13: Brechas stock y demanda docentes de educación básica según modificaciones en proporción de horas lectivas y no lectivas

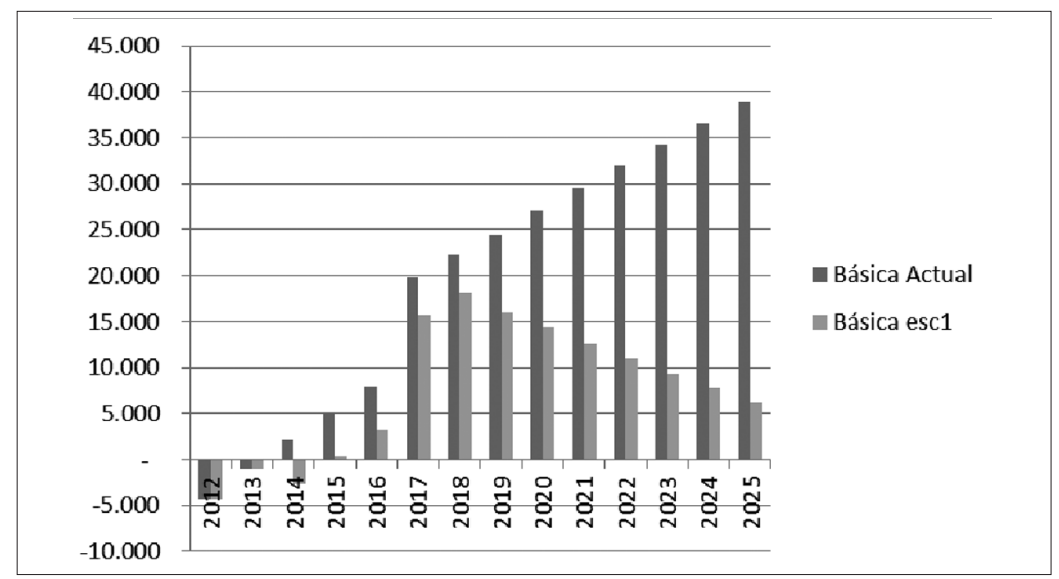

Fuente: Elaboración propia a partir de datos Unidad de Estudios Mineduc, SIES e Idoneidad Docente.

En términos agregados, los docentes de enseñanza media presentarían un superávit a lo largo de todo el periodo de estimación, con una eventual reducción de las brechas estimadas en la situación actual. A partir de 2018, y bajo el escenario de cambio, el superávit se estabilizaría alcanzando una cifra cercana a los 40.000 docentes.

Figura 14: Brechas stock y demanda docentes de educación media según modificaciones en proporción de horas lectivas y no lectivas

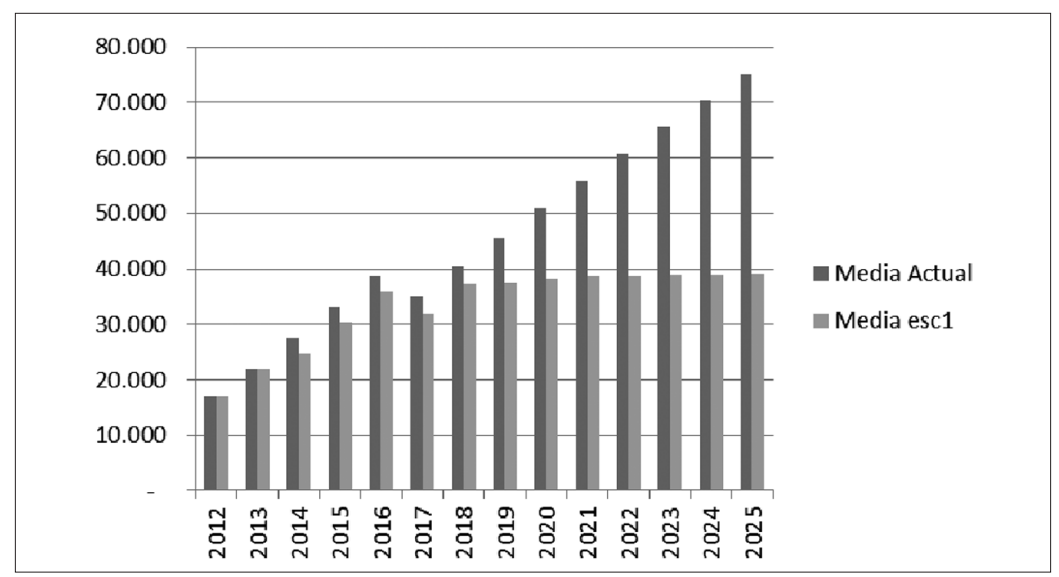

Fuente: Elaboración propia a partir de datos Unidad de Estudios MINEDUC, SIES e Idoneidad Docente. 
Al considerar los posibles efectos por macrozona, se presentaría un aumento importante de las brechas de demanda insatisfecha para la zona norte, superando los 1.000 docentes de básica no cubiertos entre 2014 y 2016. Si consideramos las condiciones bases (escenario actual), esta situación se revertiría a partir de 2017 y se mantendría hasta el final del periodo. Sin embargo, el escenario es muy distinto si se consideran las nuevas estrategias de selectividad y modificación del porcentaje de horas no lectivas, pues estas generarían una disminución del superávit a partir de 2018 e incluso presentaría un déficit de docentes a partir de 2023 que iría en aumento hasta 2025.

Figura 15: Brechas stock y demanda docentes de educación básica según modificaciones en proporción de horas lectivas y no lectivas, por macrozona, zona norte

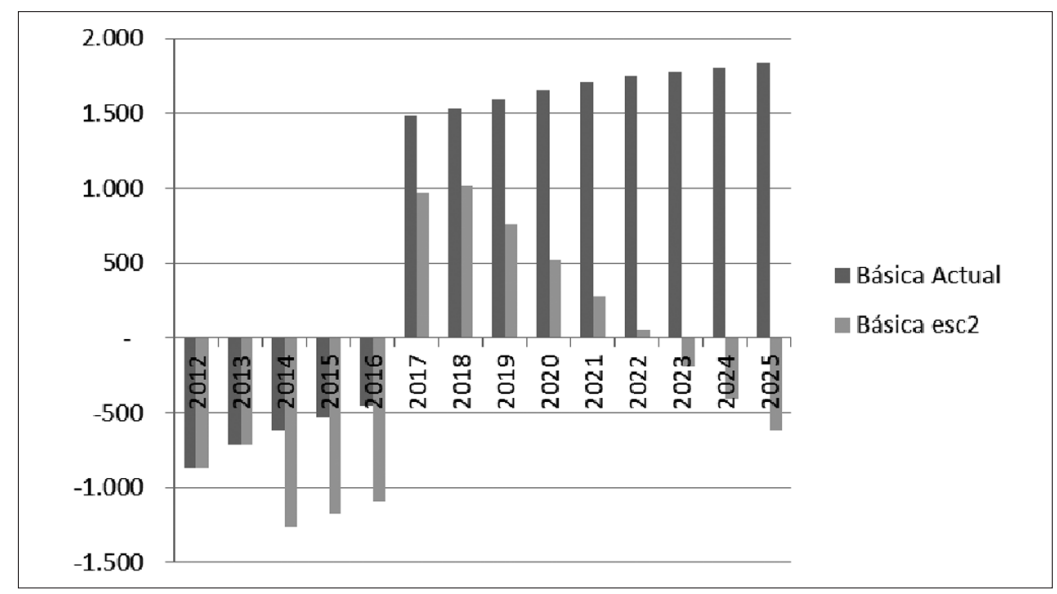

Fuente: Elaboración propia a partir de datos Unidad de Estudios Mineduc, SIES e Idoneidad Docente.

En enseñanza media, en cambio, predominaría un superávit durante todo el periodo de estimación, el que tendería a ser considerablemente más alto en las condiciones actuales. Así, mientras para el 2025 se estimaría un superávit de alrededor de 9.000 profesores en el escenario actual, este sería de alrededor de 3.500 docentes al incluirse las modificaciones. 
Figura 16: Brechas stock y demanda docentes de educación básica según modificaciones en proporción de horas lectivas y no lectivas, por macrozona, zona norte

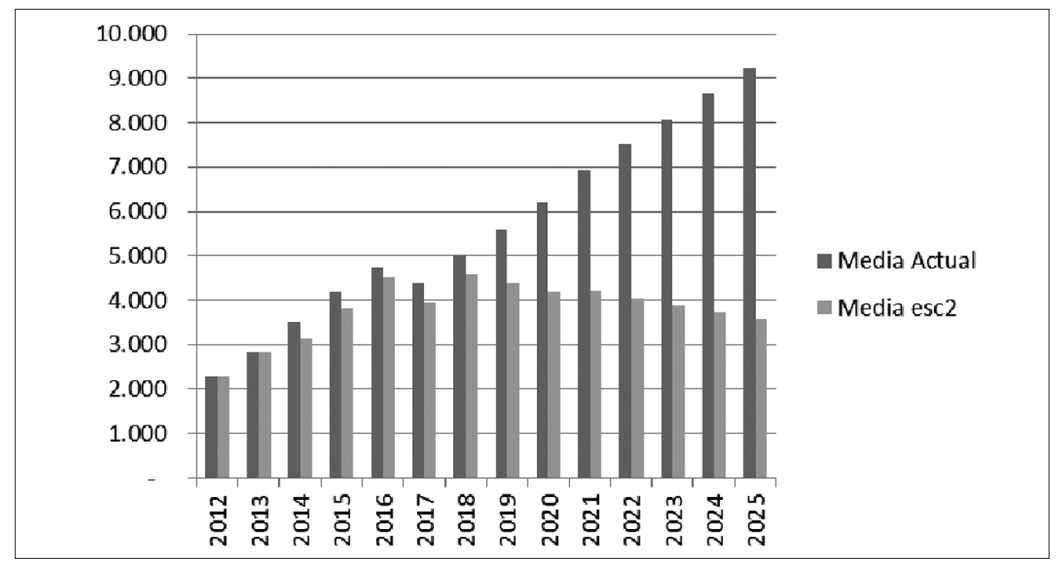

Fuente: Elaboración propia a partir de datos Unidad de Estudios Mineduc, SIES e Idoneidad Docente.

Para la zona centro se presentarían brechas insatisfechas de docentes de educación básica hasta el 2015. Sin embargo, a partir de 2016, el stock docente sí cubriría la demanda estimada con un leve superávit, dado los cambios estimados en la proporción de horas lectivas y no lectivas.

Figura 17: Brechas stock y demanda docentes de educación básica según modificaciones en proporción de horas lectivas y no lectivas, por macrozona, zona centro

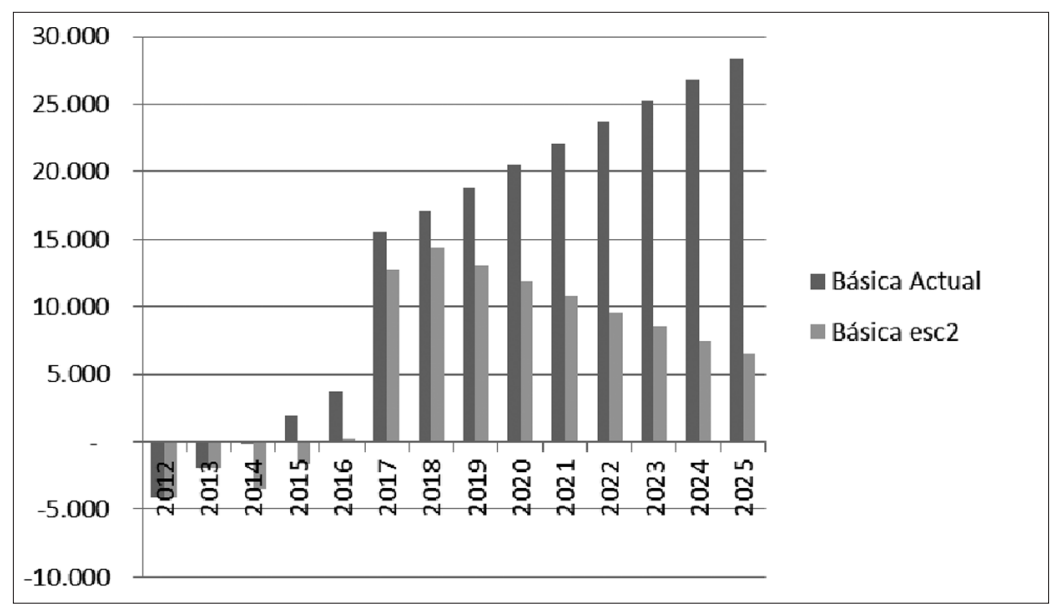

Fuente: Elaboración propia a partir de datos Unidad de Estudios Mineduc, SIES e Idoneidad Docente. 
Para los docentes de educación media, se observaría un superávit mayor a los 10.000 docentes durante los primeros años de estimación, lo que iría en ascenso bajo las condiciones actuales, pero que se estancaría alrededor de 30.000 si se implementara el nuevo escenario.

Figura 18: Brechas stock y demanda docentes de educación media, según modificaciones en proporción de horas lectivas y no lectivas, por macrozona, zona centro

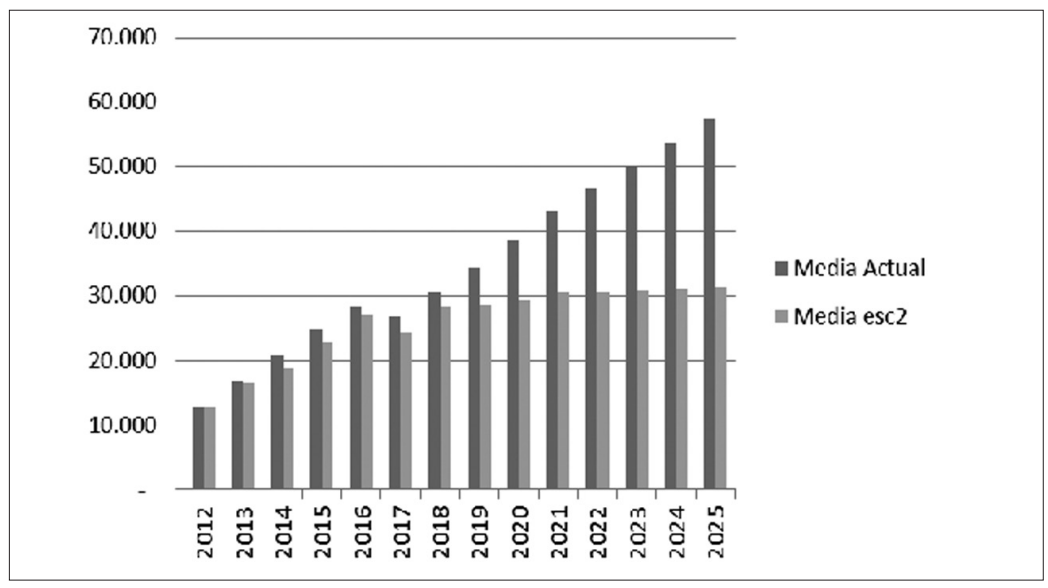

Fuente: Elaboración propia a partir de datos Unidad de Estudios Mineduc, SIES e Idoneidad Docente.

Finalmente, la zona sur presentaría un superávit anual tanto para docentes de básica como media durante todo el periodo. En el caso de enseñanza básica el superávit disminuiría en relación con el escenario actual, en el caso de implementarse el cambio en la proporción de horas no lectivas, a su vez tendería a la baja a partir de 2018 por las implicancias de la selectividad a la entrada. En enseñanza media, en cambio, la modificación en la proporcionalidad no afecta el sentido del superávit, el que permanecería creciente, sin embargo siempre se mantendría a niveles inferiores de lo que sucedería de sostenerse la situación actual. 
Figura 19: Brechas stock y demanda docentes de educación básica según modificaciones en proporción de horas lectivas y no lectivas, por macrozona, zona sur

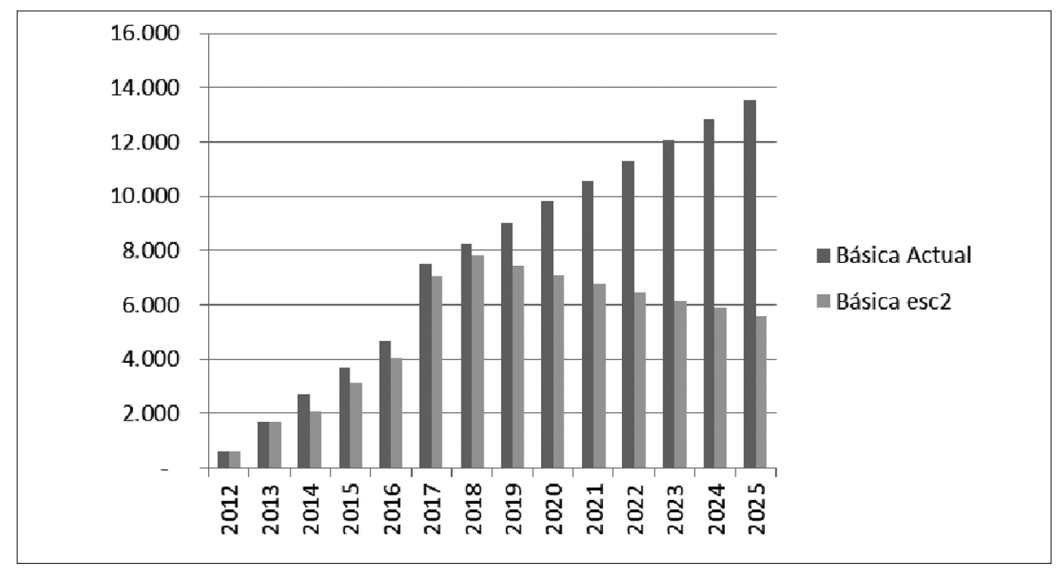

Fuente: Elaboración propia a partir de datos Unidad de Estudios Mineduc, SIES e Idoneidad Docente.

Figura 20: Brechas stock y demanda docentes de educación media según modificaciones en proporción de horas lectivas y no lectivas, por macrozona, zona sur

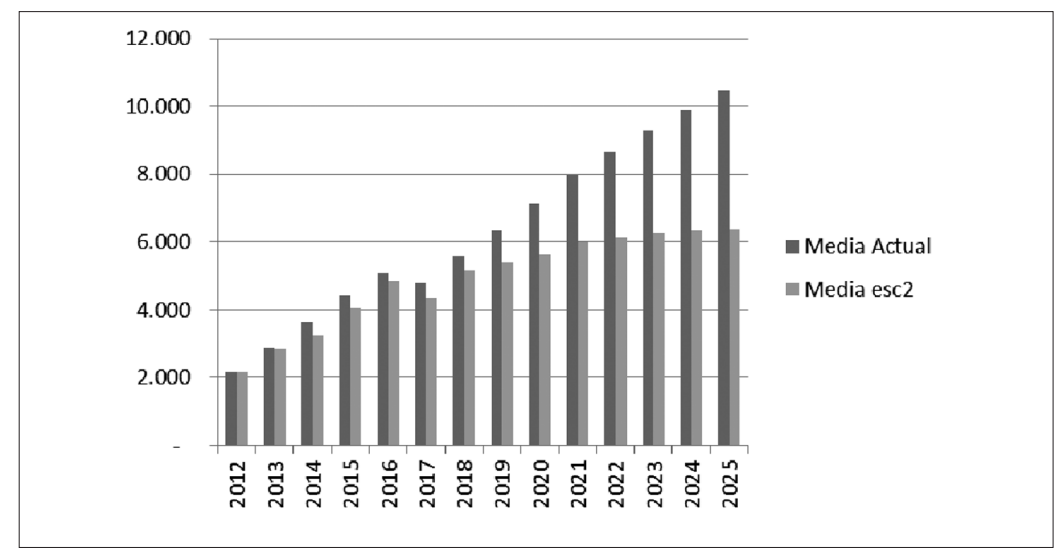

Fuente: Elaboración propia a partir de datos Unidad de Estudios Mineduc, SIES e Idoneidad Docente.

\subsection{Estimación de brechas de demanda según subsectores de aprendizaje}

De manera adicional a las estimaciones ya presentadas, se hace necesario indagar en la manera en que los nuevos criterios de selectividad para el ingreso a las carreras de educación afectarían la oferta y demanda de docentes en cada uno de los distintos subsectores de enseñanza. 
Se mantienen los supuestos presentados en el primer escenario y se estima la cantidad de titulados por especialidad asumiendo un crecimiento logarítmico de la matrícula con filtros de selección por especialidad. Porque no se cuenta con tasas de retención específicas para cada especialidad, se utilizan las tasas de retención globales asumidas anteriormente.

A nivel país, los mayores niveles de superávit se observarían en las especialidades de educación básica, inglés y educación física; especialidades que sobrepasarían los 10.000 docentes de superávit en 2025. Por el contrario, la especialidad en la que se observaría el menor superávit sería física, asignatura para la que incluso habría una demanda insatisfecha durante el periodo 2017 al 2019. Para el final el periodo de estimación, 2025, el superávit de docentes de física sería de tan solo 149, lo que representaría 16\% de superávit de demanda. Otras especialidades que también presentarían -comparativamenteun superávit bajo son química y educación artística, para las cuales el superávit de docentes al 2025 sería de 965 y 1.525, respectivamente, los que representan $85 \%$ y $53 \%$ de superávit de sus respectivas demandas.

$\mathrm{Al}$ analizar la oferta y demanda por subsectores y a nivel de macrozonas geográficas se observaría un patrón bastante similar, aunque las magnitudes varían dependiendo de la zona en cuestión. En el caso de la macrozona norte (Tabla 11), el superávit más importante se observaría en inglés y educación física, superando los 1.000 docentes en cada una de ellas y presentando la primera de estas un superávit de demanda mayor al 200\% de los requerimientos de esta especialidad. A estas especialidades les sigue historia y biología con un superávit estimado de 626 y 387 docentes para 2025, que corresponden a un superávit de demanda mayor a $100 \%$ para cada una de ellas. Por otra parte, las especialidades que presentarían menor superávit al 2025 serían matemática, con 18\%, educación artística, con $10 \%$ y educación básica, con 1,4\% de superávit de demanda. La especialidad de física presentaría una demanda no cubierta durante todo el periodo, alcanzando en 2025 42\% de demanda insatisfecha. 


\begin{tabular}{|c|c|c|c|c|c|c|c|c|c|c|c|c|}
\hline 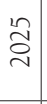 & $\begin{array}{l}\stackrel{\circ}{+} \\
\stackrel{+}{\leftarrow}\end{array}$ & $\begin{array}{l}\stackrel{0}{ }^{\circ} \\
\infty^{0} \\
\sim^{\prime}\end{array}$ & $\begin{array}{l}\stackrel{2}{a} \\
-1\end{array}$ & \begin{tabular}{l}
0 \\
\multirow{1}{1}{} \\
\multirow{1}{*}{}
\end{tabular} & $\mid \begin{array}{c}\stackrel{0}{+} \\
\stackrel{+}{0} \\
\mathfrak{\sim}^{\prime}\end{array}$ & $\begin{array}{c}\stackrel{0}{\beth} \\
\stackrel{2}{n} \\
\sim\end{array}$ & $\begin{array}{l}\infty \\
\infty \\
\delta \\
\hat{\sigma}\end{array}$ & $\begin{array}{l}0 \\
8 \\
2 \\
0 \\
-1\end{array}$ & $\begin{array}{c}\stackrel{0}{2} \\
\stackrel{3}{2} \\
0 \\
-\end{array}$ & $\begin{array}{l}\stackrel{0}{\beth} \\
\stackrel{-}{\sim} \\
\vec{\sim}\end{array}$ & 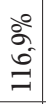 & $\begin{array}{l}20 \\
\infty \\
20 \\
1\end{array}$ \\
\hline 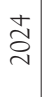 & $\begin{array}{l}\stackrel{0}{\infty} \\
\overbrace{}^{-}\end{array}$ & 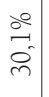 & $\begin{array}{l}\stackrel{0}{\stackrel{2}{*}} \\
\stackrel{\infty}{\infty}\end{array}$ & 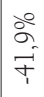 & $\mid \begin{array}{c}\dot{o}^{\circ} \\
\dot{m} \\
\dot{m}\end{array}$ & 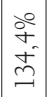 & 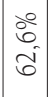 & $\mid \begin{array}{c}0 \\
\stackrel{2}{2} \\
\stackrel{2}{0}\end{array}$ & $\mid \begin{array}{c}\circ \\
\stackrel{\sim}{\beth} \\
=\end{array}$ & $\mid \begin{array}{l}0^{0} \\
\stackrel{0}{6} \\
\stackrel{-}{\sim}\end{array}$ & $\begin{array}{l}\stackrel{\circ}{2} \\
\stackrel{2}{\Xi} \\
ٍ\end{array}$ & $\begin{array}{l}\stackrel{0}{\circ} \\
\hat{1} \\
1\end{array}$ \\
\hline$\underset{\widetilde{\sim}}{\tilde{\sim}}$ & 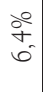 & $\begin{array}{l}\stackrel{0}{b} \\
\vec{m}\end{array}$ & 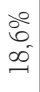 & 害 & $\left|\begin{array}{c}0 \\
\infty \\
m \\
m\end{array}\right|$ & $\mid \begin{array}{c}\stackrel{0}{2} \\
\stackrel{+}{+} \\
\stackrel{+}{+} \\
\dot{+}\end{array}$ & $\mid \begin{array}{c}0 \\
\infty \\
\infty \\
0 \\
0\end{array}$ & $\mid \begin{array}{c}\stackrel{0}{2} \\
\stackrel{2}{0} \\
ٍ\end{array}$ & $\mid \begin{array}{c}0 \\
\infty \\
\infty \\
-1\end{array}$ & $\mid \begin{array}{l}\stackrel{0}{2} \\
\stackrel{-}{-} \\
\sim \\
\sim\end{array}$ & 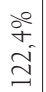 & 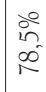 \\
\hline$\underset{\widetilde{్}}{\widetilde{\mho}}$ & $\begin{array}{l}\stackrel{\circ}{2} \\
\tilde{\sigma}^{2}\end{array}$ & $\begin{array}{l}\stackrel{2}{~} \\
\text { సे }\end{array}$ & $\begin{array}{l}\infty \\
\infty \\
\infty \\
\infty \\
-1\end{array}$ & 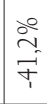 & 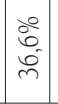 & 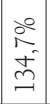 & $\mid$\begin{tabular}{l}
$\stackrel{0}{\circ}$ \\
\multirow{f}{b}{}
\end{tabular} & $\begin{array}{l}\stackrel{0}{2} \\
\stackrel{\Xi}{ \pm} \\
=\end{array}$ & $\left|\begin{array}{c}0 \\
\dot{0} \\
\\
-1\end{array}\right|$ & $\mid \begin{array}{l}0 \\
\infty \\
\infty \\
\\
\sim\end{array}$ & $\begin{array}{l}\circ \\
\infty \\
\stackrel{\sim}{\beth} \\
ٍ\end{array}$ & 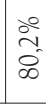 \\
\hline $\overrightarrow{\widetilde{\Omega}}$ & $\begin{array}{l}\text { ठ̊ } \\
\text { తn }\end{array}$ & 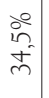 & $\begin{array}{l}\stackrel{\circ}{2} \\
\stackrel{a}{a}\end{array}$ & $\begin{array}{l}\text { 足 } \\
\text { in } \\
\text { ơ }\end{array}$ & $\left|\begin{array}{l}0 \\
0 \\
0 \\
\delta \\
\tilde{m}\end{array}\right|$ & $\mid \begin{array}{l}\stackrel{0}{1} \\
\underset{b}{0} \\
\check{n}\end{array}$ & 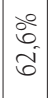 & $\mid \begin{array}{c}0 \\
\infty \\
0 \\
0 \\
= \\
=\end{array}$ & $\mid \begin{array}{l}0 \\
\stackrel{2}{1} \\
\\
-1\end{array}$ & $\mid \begin{array}{c}\circ \\
\stackrel{0}{2} \\
\infty^{\prime} \\
\vec{\sim}\end{array}$ & 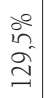 & $\begin{array}{l}0 \\
\text { ò } \\
\text { ¿ }\end{array}$ \\
\hline 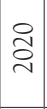 & $\begin{array}{l}\stackrel{0}{\circ} \\
\dot{J}^{\prime}\end{array}$ & $\underset{\stackrel{F}{\rightleftharpoons}}{\stackrel{\circ}{二}}$ & $\begin{array}{l}\stackrel{0}{\stackrel{2}{m}} \\
\stackrel{\sim}{\sim}\end{array}$ & 今̊ & $\begin{array}{l}\stackrel{\circ}{i} \\
\tilde{f}\end{array}$ & $\mid \begin{array}{c}0 \\
\stackrel{2}{a} \\
\beth \\
=\end{array}$ & $\mid \begin{array}{l}\stackrel{0}{2} \\
\stackrel{1}{1} \\
\infty \overbrace{}^{2}\end{array}$ & $\mid \begin{array}{l}\circ \\
\frac{a}{q} \\
a\end{array}$ & $\mid$\begin{tabular}{l}
$\stackrel{0}{2}$ \\
\multirow{2}{n}{} \\
in
\end{tabular} & $\mid \begin{array}{c}\stackrel{\circ}{+} \\
\stackrel{+}{i} \\
\stackrel{i}{N}\end{array}$ & $\begin{array}{l}\circ \\
\stackrel{0}{N} \\
\end{array}$ & $\begin{array}{l}\stackrel{0}{2} \\
\stackrel{2}{2}\end{array}$ \\
\hline$\stackrel{\vec{\nu}}{\vec{\nu}}$ & $\begin{array}{l}\stackrel{0}{2} \\
\text { ડ- } \\
-1\end{array}$ & $\begin{array}{l}\stackrel{\circ}{i n} \\
\tilde{f}\end{array}$ & $\begin{array}{l}\stackrel{\circ}{+} \\
\stackrel{\sim}{\sim}\end{array}$ & 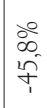 & $\begin{array}{l}\stackrel{\circ}{i n} \\
\dot{m}\end{array}$ & $\mid \begin{array}{c}0 \\
\stackrel{0}{\sim} \\
ٍ \\
=\end{array}$ & $\begin{array}{l}\gtrless^{\circ} \\
\frac{1}{i}\end{array}$ & $\begin{array}{l}\stackrel{0}{m_{2}^{2}} \\
\tilde{n}^{2}\end{array}$ & $\mid \begin{array}{c}\circ \\
\dot{y} \\
i \\
i n\end{array}$ & $\begin{array}{l}\stackrel{0}{2} \\
\tilde{2} \\
\tilde{2}\end{array}$ & 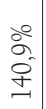 & $\stackrel{\stackrel{\circ}{\sim}}{\stackrel{\infty}{\infty}}$ \\
\hline$\stackrel{\infty}{\stackrel{\sim}{二}}$ & $\begin{array}{l}\stackrel{\circ}{\stackrel{\sim}{\sim}} \\
\stackrel{\sim}{二}\end{array}$ & $\left|\begin{array}{c}0 \\
8 \\
f \\
f \\
0\end{array}\right|$ & 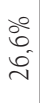 & 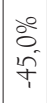 & $\begin{array}{l}\stackrel{\circ}{\sim} \\
\stackrel{5}{m}\end{array}$ & 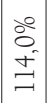 & 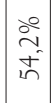 & $\begin{array}{l}\text { 亏̊ } \\
\dot{H}^{2}\end{array}$ & $\mid \begin{array}{l}0 \\
0 \\
8 \\
8\end{array}$ & 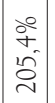 & 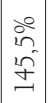 & $\begin{array}{l}\stackrel{0}{\leftarrow} \\
\stackrel{\infty}{\infty}\end{array}$ \\
\hline$\stackrel{\vec{\sim}}{\vec{\sim}}$ & 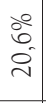 & $\mid \begin{array}{c}\circ \\
\stackrel{f}{f}\end{array}$ & $\begin{array}{l}\stackrel{\circ}{\sim} \\
\stackrel{\leftarrow}{\sim}\end{array}$ & 竞 & 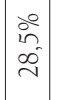 & 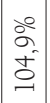 & 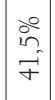 & 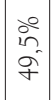 & 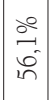 & $\begin{array}{c}0 \\
0 \\
0 \\
01 \\
0 \\
-1\end{array}$ & 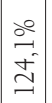 & $\frac{\stackrel{\circ}{+}}{\stackrel{n}{r}}$ \\
\hline $\begin{array}{l}0 \\
\stackrel{2}{\sim} \\
\stackrel{\sim}{*}\end{array}$ & 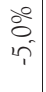 & $\mid \begin{array}{c}0 \\
\stackrel{o}{+} \\
o \\
\ddots \\
=\end{array}$ & \begin{tabular}{l}
$\stackrel{8}{0}$ \\
\multirow{\infty}{\infty}{} \\
$\infty$
\end{tabular} & 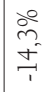 & $\mid \begin{array}{l}0 \\
\dot{0} \\
0 \\
0\end{array}$ & 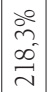 & $\left|\begin{array}{c}\stackrel{0}{+} \\
\dot{+} \\
\infty \\
m\end{array}\right|$ & $\begin{array}{l}0 \\
\stackrel{0}{\tilde{\sigma}} \\
\tilde{\sigma}\end{array}$ & $\mid$\begin{tabular}{c}
$\circ$ \\
$\stackrel{+}{a}$ \\
$ٌ$ \\
\hdashline
\end{tabular} & 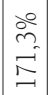 & $\mid \begin{array}{c}0 \\
8 \\
0 \\
8 \\
0\end{array}$ & $\frac{\circ}{2}$ \\
\hline$\stackrel{\stackrel{20}{\circ}}{\stackrel{2}{\sim}}$ & $\begin{array}{l}\stackrel{\circ}{2} \\
\stackrel{1}{1}\end{array}$ & $\mid \begin{array}{c}0 \\
\grave{2} \\
8 \\
8\end{array}$ & $\begin{array}{l}\stackrel{\circ}{+} \\
\stackrel{\infty}{1}\end{array}$ & $\begin{array}{l}\stackrel{0}{\circ} \\
\infty 0^{\prime} \\
1\end{array}$ & $\begin{array}{l}\stackrel{0}{\sigma} \\
\hat{\infty} \\
\infty\end{array}$ & 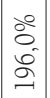 & $\left|\begin{array}{l}20 \\
\stackrel{2}{0} \\
\infty \\
-1\end{array}\right|$ & $\mid \begin{array}{l}0 \\
8 \\
8 \\
8\end{array}$ & $\left|\begin{array}{l}0 \\
0 \\
\vdots \\
\pm\end{array}\right|$ & 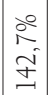 & $\left|\begin{array}{c|}\stackrel{0}{2} \\
i \\
\infty \\
1 \\
10\end{array}\right|$ & 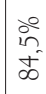 \\
\hline$\underset{\sim}{\stackrel{\vec{V}}{\mathbf{D}}}$ & $\begin{array}{l}\text { مे } \\
\hat{\rho} \\
1\end{array}$ & $\left|\begin{array}{c}0 \\
\infty \\
\vdots \\
\sigma\end{array}\right|$ & $\stackrel{\stackrel{2}{2}}{\stackrel{N}{N}}$ & 官 & 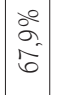 & 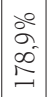 & $\begin{array}{l}20 \\
2 \\
6\end{array}$ & $\mid \begin{array}{l}2^{\circ} \\
i^{0} \\
0^{0}\end{array}$ & $\begin{array}{l}a_{2}^{0} \\
\tilde{a}\end{array}$ & $\mid \begin{array}{c}\stackrel{\circ}{+} \\
+ \\
\pm \\
\pm \\
=\end{array}$ & $\mid \begin{array}{l}\stackrel{0}{2} \\
\stackrel{2}{1} \\
i n \\
i n \mid\end{array}$ & $\begin{array}{l}\stackrel{8}{\circ} \\
\dot{0}\end{array}$ \\
\hline 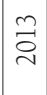 & $\begin{array}{l}\delta_{0}^{0} \\
\infty_{1}^{-} \\
1\end{array}$ & $\mid$\begin{tabular}{c|}
$\stackrel{0}{2}$ \\
$\frac{1}{\infty}$ \\
$\infty$
\end{tabular} & $\frac{\circ}{8^{\circ}}$ & 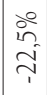 & $\begin{array}{l}i 0 \\
i n \\
i n\end{array}$ & $\mid \begin{array}{c}\stackrel{0}{2} \\
\stackrel{\tilde{J}}{\sigma} \\
0\end{array}$ & $\begin{array}{l}\circ \\
\stackrel{0}{1} \\
\hat{1}\end{array}$ & $\begin{array}{l}0 \\
i \mathfrak{1} \\
6\end{array}$ & \begin{tabular}{l}
$\stackrel{2}{\sigma}$ \\
\multirow{\sigma}{*}{}
\end{tabular} & $\mid \begin{array}{l}0^{\circ} \\
1- \\
\infty \\
\infty\end{array}$ & $\left|\begin{array}{l}\stackrel{0}{2} \\
i n \\
\infty \\
m \\
m\end{array}\right|$ & $\begin{array}{l}\text { oे } \\
\text { ó } \\
\text { in }\end{array}$ \\
\hline 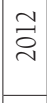 & $\begin{array}{l}\overbrace{}^{\circ} \\
i^{n}\end{array}$ & $\left|\begin{array}{c}\partial^{\circ} \\
\infty^{-} \\
1\end{array}\right|$ & $\begin{array}{l}\stackrel{\circ}{\circ} \\
\hat{0}\end{array}$ & 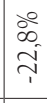 & $\left|\begin{array}{c}\circ \\
\vdots \\
j \\
f\end{array}\right|$ & $\mid \begin{array}{c}\stackrel{0}{2} \\
i n \\
i n \\
\end{array}$ & $\begin{array}{l}\stackrel{0}{\stackrel{+}{t}} \\
\stackrel{1}{1}\end{array}$ & $\begin{array}{l}\stackrel{\circ}{2} \\
\dot{0} \\
\tilde{8}\end{array}$ & $\mid \begin{array}{c}\stackrel{\circ}{+} \\
\stackrel{-}{\tau}\end{array}$ & $\begin{array}{l}0 \\
6 \\
6 \\
6\end{array}$ & $\begin{array}{l}\stackrel{0}{2} \\
2 \\
2\end{array}$ & $\begin{array}{l}\text { o̊ } \\
\text { in }\end{array}$ \\
\hline 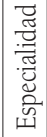 & 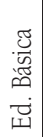 & 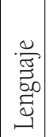 & 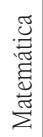 & 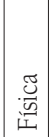 & 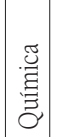 & 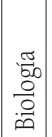 & 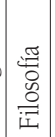 & 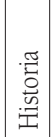 & 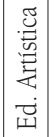 & 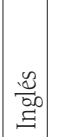 & 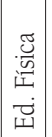 & $\stackrel{. \pi}{\frac{\pi}{\sigma}}$ \\
\hline
\end{tabular}


Para la macrozona centro en términos generales se presentaría un superávit en todas las especialidades con excepción de física para el periodo 2017-2020 (Tabla 12). El mayor superávit lo presentarían las especialidades de inglés y filosofía superando el 200\% de demanda satisfecha. Le siguen educación física e historia con un superávit de demanda mayor a $150 \%$. Las especialidades que presentarían un superávit más reducido son educación artística, educación básica y física, todas por debajo del $40 \%$ de superávit de sus respectivas demandas. 


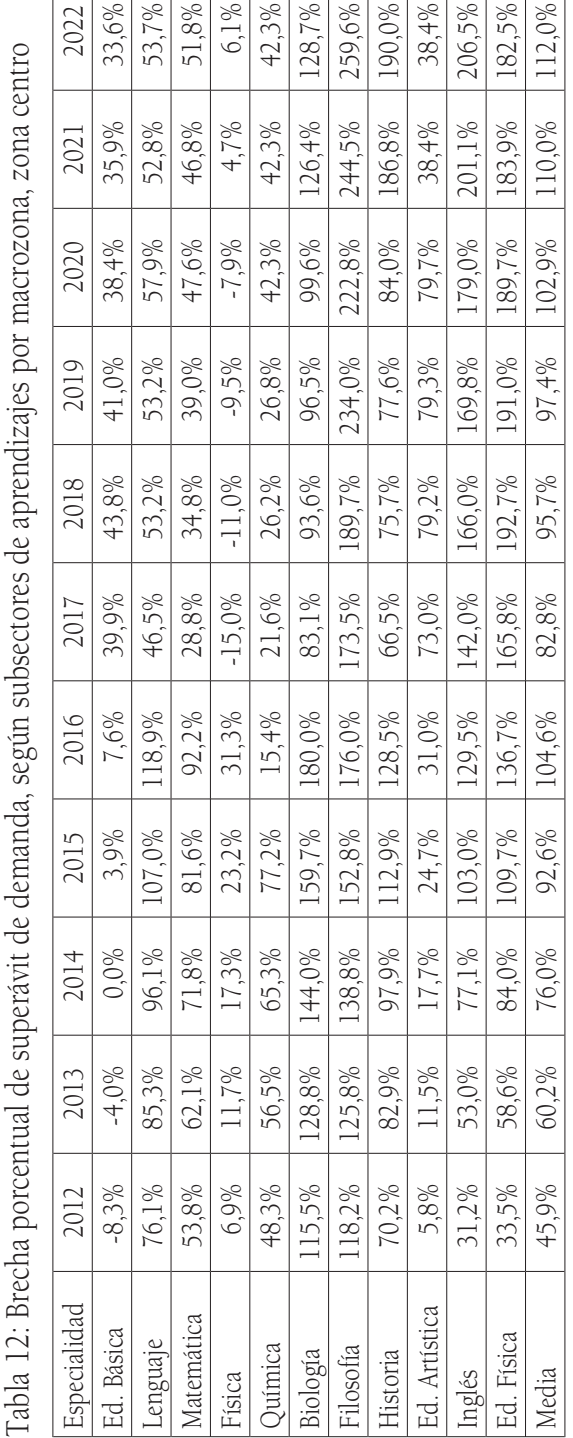

ป̂ ปิ ปิ

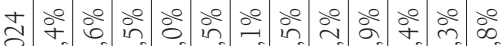
ปิ حิ

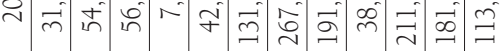
તี่

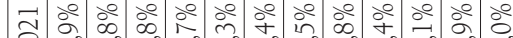

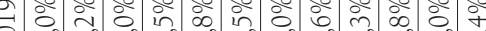

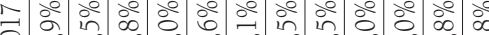

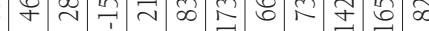

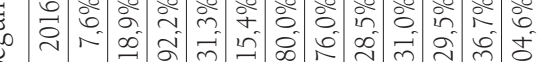

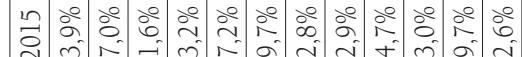

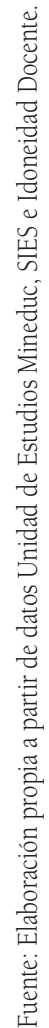


Finalmente para la macrozona sur se observaría una brecha de demanda no cubierta para las especialidades de física y educación artística para casi todo el periodo de estimación, alcanzando en 2025 casi $10 \%$ de demanda insatisfecha para ambas especialidades (Tabla 13). El mayor superávit estimado lo presentarían las especialidades de inglés, educación física, historia y biología, todas con más de 200\% de superávit de demanda. Por su parte la especialidad de química solo alcanzaría 30\% de superávit para el 2025, presentando entre 2016 y 2018 una brecha de demanda insatisfecha.

\section{Limitaciones}

Es necesario tener algunas consideraciones a la hora de interpretar los hallazgos de esta investigación. En primer lugar es relevante señalar que la proyección de la demanda y su comparación con la proyección del stock se realizó a partir de los datos de los docentes que se encontraban desempeñando trabajo de aula en establecimientos urbanos. Estos docentes eran 153.119 y representaban el $72,95 \%$ de los profesores que se encontraban trabajando en establecimientos educacionales del país.

Junto con lo anterior, al proyectar el stock de docentes se estableció como base las cifras de 2011, considerando a los docentes en ejercicio y a los titulados ese año. Dicha estimación es conservadora, pues no considera a todos los docentes que tienen título de profesor y que actualmente no se encuentran trabajando como tales.

Al proyectar la cantidad de docentes según puntaje PSU, se considera la incorporación al mercado laboral únicamente de los alumnos titulados en cinco años, sin acumular a los alumnos no titulados al siguiente periodo. Se destaca por tanto, que la estimación realizada subestima la cantidad de nuevos docentes en ejercicio.

Por otro lado, es necesario mencionar que existen requerimientos de docentes de media, superiores a los presentados en la sección de demanda, pues por currículo a las horas de enseñanza general media no se le asigna a una especialidad particular e incluyen horas de orientación y otros. Lo anterior podría disminuir levemente las brechas de stock y demanda docente a nivel agregado. 


\section{Conclusiones y propuestas}

Uno de los principales cambios que ha experimentado el sistema educativo nacional en los últimos años ha sido la modificación de su composición, caracterizada por una reducción en la matrícula escolar total (con un pronunciado énfasis en la disminución en el sector municipal) y un aumento sostenido de los titulados en carreras de educación. Considerando esta información y la disminución de la tasa de natalidad de Chile es posible inferir que el mercado docente presentará algún tipo de desajuste en el corto plazo.

Al proyectar el comportamiento de la demanda docente de nuestro sistema y del stock disponible para satisfacerla, fue posible corroborar la hipótesis del desajuste. Las estimaciones realizadas muestran una tendencia de disminución de la demanda docente en la mayor parte de los niveles, especialidades, dependencias y zonas geográficas analizadas. A la vez, se observó un gran crecimiento del stock de profesionales con título en educación.

En términos agregados, se presentaría un superávit importante de docentes en la mayoría de las especialidades de enseñanza media, sobre todo en el caso de biología, filosofía y lenguaje. Las únicas excepciones serían los casos de educación general básica y física, que presentarían déficit para la mayoría de los años proyectados.

A nivel de zonas geográficas, se registra un importante superávit de profesores para cada una de ellas en las especialidades de inglés y educación física. Aunque la tendencia general por niveles y especialidades en el territorio es de superávit de profesores, se identifican excepciones como la especialidad de física que presenta déficit en todo el territorio.

La experiencia internacional revisada en el marco de este estudio sugiere que el superávit de profesores se asocia a una baja calidad de docentes y a una baja en la valorización social de la misma. Debido a lo anterior, los datos acerca del agudo superávit actual en el país sugieren la necesidad de desarrollar reformas que limiten la cantidad de profesores en el sistema, especialmente en relación con las condiciones para ingresar a carreras de educación. Estas medidas, 
sin embargo, deberían implementarse considerando las excepciones asociadas a los territorios y especialidades.

Como parte de los análisis de esta investigación, se simularon escenarios de implementación de políticas de mayor selectividad para el ingreso a carreras de educación, así como también modificaciones en la proporción de horas lectivas/no lectivas de los profesores. La aplicación conjunta de mayores exigencias en el ingreso a carreras de educación (basadas en puntajes PSU y ranking de notas) y disminución del tiempo lectivo de profesores contribuiría a aminorar el superávit de docentes tanto en enseñanza básica como media. De esta forma, el superávit de docentes de educación básica se estabilizaría en 2015 en cerca de 5.000 para alcanzar una cifra cercana a los 23.000 en 2018, y luego comenzar a reducir la brecha si se implementaran las políticas consideradas. Para las especialidades de educación media, en 2015 se tendría un superávit agregado mayor a 30.000 docentes, alcanzando una cifra superávit de aproximadamente 40.000 en 2018. Sin embargo, el nivel de superávit no se distribuye de manera uniforme en el territorio nacional. Así, la zona norte presentaría déficit de profesores de educación básica a partir de 2023, mientras que la zona sur mantendría un superávit más leve que el actual tanto en la enseñanza básica como en la media. Aunque a nivel nacional las medidas analizadas tienden a disminuir el superávit que actualmente existe en la mayor parte de las especialidades, el análisis por zonas geográficas alerta acerca de la desigual distribución de profesores, registrándose déficits en ciertas asignaturas en periodos específicos de la estimación en las zonas norte y sur.

Porque el superávit de profesores se asocia con problemas de calidad y valoración social de la profesión, resulta necesario analizar alternativas que permitan, de forma cuidadosa, disminuir las importantes tasas de superávit que se registran en el sistema nacional. En este sentido, además de las medidas analizadas en este estudio, conviene considerar otras que se han implementado en países con dificultades similares: licitar un número reducido de programas de formación (de alta selección y exigencia), limitar el número de profesores que se puede formar en cada área o establecer exigencias elevadas para acceder a programas de formación. 
Complementariamente, el uso de un examen de habilitación al final de la carrera podría vincularse con el mismo propósito. Más allá del camino seleccionado, resulta relevante que los encargados de tomar decisiones avancen en establecer mecanismos que impidan el creciente superávit de profesores, especialmente si presentan bajas calificaciones.

Por otro lado, es imprescindible estimular el retiro de profesores considerados inefectivos, de modo que los nuevos titulados que cuenten con altas calificaciones puedan ejercer la profesión en que se han formado y, de esta forma, incrementar los niveles de calidad en el sistema.

Como se mencionó anteriormente, este estudio solo ha abordado a aquellos profesores que ejercen en el ciclo básico y medio en establecimientos de zonas urbanas que se desempeñan en el aula. Así, resultaría muy importante considerar estimaciones similares que incorporen otras variables importantes. En primer lugar, resultaría relevante contar con información para el área de la educación parvularia, que ha experimentado un explosivo aumento de estudiantes en los últimos años, y en la que ha existido un aumento significativo en la cobertura. En segundo lugar, sería importante incluir estimaciones para el sector rural, cuyo caso podría diferir de manera importante con las conclusiones señaladas en este documento. Finalmente, debería profundizarse en la distribución de docentes según zonas geográficas. Eventualmente, las cifras agregadas podrían estar ocultando severos déficits en algunas regiones y un incluso más importante superávit en otras.

\section{Referencias bibliográficas}

Barber, M., y M. Mourshed, (2007) How the word's best - performing school systems come out on top. Nueva York, McKinsey \& Company.

Cooper, J. y A. Alvarado, (2006) Preparation, recruitment, and retention of teachers. Paris, Educational Policy Series. International Institute for Education Planning, UNESCO.

Elacqua, G., (2012) "The impact of school choice and public policy on segregation: Evidence from Chile", en International Journal of Educational Development 32, pp. 444-453. 
Hanushek, E., (2011) "The economic value of higher teacher quality", en Economics of Education Review, Vol 30, Issue 3, junio 2011, pp. 466-479.

Ingersoll, R., (2007) "A comparative study of teacher preparation and qualifications in six nations". CPRE Policy Briefs, febrero 2007.

Montoya, A., y S. Blackburn, (2010) Estudio de oferta y demanda de docentes en Chile. Proyección 2010-2018. Santiago: Centro de Perfeccionamiento, Experimentación e Investigaciones Pedagógicas del Ministerio de Educación.

OECD, (2005) Teachers matters: attracting, developing and retaining effective teachers. Paris, OECD.

Rockoff, J., (2004) The impact of individual teachers on student achievement: evidence from panel data. Papers and proceedings. American Economic Review Vol. 94, N² 2, pp. 247-252.

Sanders, W. y J. Rivers, (1996) Cumulativve and residual effects of teachers on future student academic achievement. Tennessee, Knoxville, University of Tennessee, Value-Added Research and Assessment Center.

Santiago, P., (2002) "Teacher demand and supply: improving teaching quality and addressing teacher shortages". Paris, OECD Education Working Papers, $\mathrm{N}^{\circ}$. 1, OECD Publishing.

Vegas, E., (2007) "Teacher labor markets in developing countries", en The future of children, Vol. 17, № 1, primavera 2007, pp. 219-232.

Recibido: 28/03/2013

Aceptado: 03/11/2013 